\title{
Non-centralized Control for Flow-based Distribution Networks: A Game-theoretical Insight
}

\author{
Julian Barreiro-Gomez ${ }^{\mathrm{a}, \mathrm{b}}$, Carlos Ocampo-Martinez ${ }^{\mathrm{a}}$, Nicanor Quijano ${ }^{\mathrm{b}}$, and \\ Jose M. Maestre ${ }^{\mathrm{c}}$ \\ ${ }^{a}$ Automatic Control Department, Universitat Politècnica de Catalunya, Institut de Robòtica i Informàtica Industrial \\ (CSIC-UPC), Llorens i Artigas, 4-6, 08028 Barcelona, Spain \\ ${ }^{\mathrm{b}}$ Departamento de Ingeniería Eléctrica y Electrónica, Universidad de los Andes, Carrera 1 No 18A-10, Bogotá, Colombia \\ ${ }^{\mathrm{c}}$ Departamento de Ingeniería de Sistemas y Automática, Universidad de Sevilla, 41092 Sevilla, Spain
}

\begin{abstract}
This paper solves a data-driven control problem for a flow-based distribution network with two objectives: a resource allocation and a fair distribution of costs. These objectives represent both cooperation and competition directions. It is proposed a solution that combines either a centralized or distributed cooperative game approach using the Shapley value to determine a proper partitioning of the system and a fair communication cost distribution. On the other hand, a decentralized noncooperative game approach computing the Nash equilibrium is used to achieve the control objective of the resource allocation under a non-complete information topology. Furthermore, an invariant-set property is presented and the closed-loop system stability is analyzed for the non-cooperative game approach. Another contribution regarding the cooperative game approach is an alternative way to compute the Shapley value for the proposed specific characteristic function. Unlike the classical cooperative-games approach, which has a limited application due to the combinatorial explosion issues, the alternative method allows calculating the Shapley value in polynomial time and hence can be applied to large-scale problems.
\end{abstract}

Key words: Flow-based distribution networks, population games, Nash equilibrium, cooperative games, Shapley value, dynamic resource allocation, partitioning approach, distributed control.

\section{Introduction}

The ideal centralized control scheme in which a single entity governs a system using full information, disposing of enough time to gather all the measurements, and to compute and transmit all the control inputs, may not be realistic for certain systems. Real large-scale control problems usually present issues that limit the application of centralized control strategies, e.g., fast dynamics with demanding response times, unavailability of full information, intractability of the problem due to its sheer size, among others. These issues are of special interest in the control field and a great effort has been placed into the development of non-centralized control techniques in order to mitigate them. In addition, significant technological changes have occurred during the last decades that have modified the way these problems are addressed. This is particularly visible if the methods used some decades ago [34] are compared with those used today [17]. Nowadays, computing and networking elements are pervasive and state-of-the-art control techniques are more and more sophisticated to take advantage of the new possibilities that technology offers.

This paper focuses on one of the recent trends in the development of non-centralized control techniques. In particular, during the last years different control strategies, which are able to adapt dynamically to the evolution of the system

Email addresses: j.barreiro135@uniandes.edu.co - jbarreiro@iri.upc.edu (Julian Barreiro-Gomez), cocampo@iri.upc.edu (Carlos Ocampo-Martinez), nquijano@uniandes.edu.co (Nicanor Quijano), pepemaestre@us.es (Jose M. Maestre). 
under control and its structure, have been presented. For example, in [25] it is proposed a suitable on-line method to decide which information should be shared and used between different local predictive controllers operating with various topologies. In [33], plug-and-play decentralized model predictive controllers are studied. In [14], a method to design simultaneously the communication topology and feedback control laws is proposed. Likewise in [18], a control method that switches between different linear feedback controllers to attain a trade-off between communication burden and performance is proposed. This latter work has been extended to the model predictive framework in [7]. The rationale of this work is somehow similar to [18] since the control architecture changes its topology in order to reduce the communication burden. However, unlike the aforementioned works, the control strategy proposed in this work uses both a cooperative and a non-cooperative game-theory approaches. Game theory has become a powerful tool in the design of learning and optimization-based systems [4][19][31]. Both cooperative and non-cooperative game approaches have been widely used in the design of controllers depending on their control objectives. In some cases, it is more suitable to work with a cooperative perspective when agents can collaborate among them, whereas there are other situations in which it is more appropriate to state the problem as a competition. However, there are control problems that integrate both cooperation and competition in two different stages. Furthermore, the proposed approach is a data-driven control inspired by the dynamic resource allocation problem [1][29].

Another contribution of this paper is to deepen into the relationship between game theory and control. Being the mathematical field that deals with situations of mutual interaction [22], game theory has a natural application in the context of non-centralized controllers. Broadly speaking, game theory has two main branches depending on whether the players of the game are capable to cooperate with each other or not. In particular, non-cooperative game theory deals with problems where players make decisions to maximize their utilities. A typical outcome of a non-cooperative game is given by the Nash equilibrium, condition in which no player can improve their utilities without the detriment of the utility of other players. In order to solve the non-cooperative game, it is proposed the use of population games, e.g., replicator, projection, Smith, Brown-Von-Neumann-Nash, best response, and logit choice dynamics [35]. In contrast, cooperative game theory studies the conditions and payoff rules for groups of players that form coalitions. A solution of a cooperative game is given by the Shapley value, which assigns a fair payoff to each player according to its contribution. There are several control solutions that use both game theory approaches. For instance in [16], an application of non-cooperative game theory can be found, where a distributed control strategy based on the convergence to a Nash equilibrium is proposed [24]. Furthermore, convergence to Nash equilibrium by using evolutionary-game theory has been used in the design of control and optimization strategies [3][26][39]. On the other hand, cooperative game theory has been used for example in [6], where a coalitional control approach is introduced, or in [18], where a control scheme considering different network topologies is presented. In this and other related works such as [21], the links that compose the network topology are transformed into the players of a game and the payoff given by the Shapley value is used as a mean to determine the relevance of the links [37]. Other works that mainly use the Shapley value are [10], and [15]. In [10] the Shapley value as a distribution rule is used to guarantee the existence of a Nash equilibrium in any game. In [15] an evolutionary coalitional approach is proposed so that entities can decide in an autonomous manner whether it is profitable or not to make a coalition.

Several engineering problems may be addressed as a flow-based distribution network as discussed in [11]. In this paper, a specific flow-based distribution network is considered, and a combination of two game-theoretical approaches is used to propose a decentralized control strategy (i.e., population and cooperative games). The incentives of the aforementioned combination are given by the fact that the control problem involves both competition and cooperation in two different objectives, i.e., competition in a dynamical resource allocation problem, and cooperation in the fair distribution of communication costs. More specifically, the flow-based distribution network is divided into different partitions. These partitions are determined by a criterion based on a cooperative game, i.e., by using the Shapley value. Unlike [20], where the Shapley value is computed by considering weights of the links in a complete graph, this paper proposes the use of the error with respect to the maximum capacity of storage elements in the network. Furthermore, a population-game approach is implemented at each partition to solve the resource allocation problem without using a complete information topology. Then, a data-driven dynamic resource allocation problem is solved at each sub-system by converging to a Nash equilibrium. The stability of the closed-loop system, composed of the flow-based distribution network and the population games, is analyzed by using passivity theory as in [1][8][30][32]. Moreover, this paper proposes a different way to compute the Shapley value for a specific characteristic function in the cooperative game in order to reduce the computational burden, which is one of the main issues when using this game-theoretical approach. Unlike the classical cooperative-games approach, which has a limited application due to the combinatorial explosion issues, the alternative method allows calculating the Shapley value in polynomial time and hence can be applied to large-scale problems. Besides, the considerable reduction in the computational cost also allows computing the Shapley value in a distributed way. Finally, in order to show the performance of the proposed methodology based on cooperative and non-cooperative games, a resource allocation problem in a water system treated in [32] is presented. Different from the preliminary work presented in [2], this paper includes analysis 
regarding invariant-set properties that guarantee a limitation resource constraint, and the stability of the whole closed-loop system. The resource allocation problem has been addressed with replicator and projection dynamics since they share some important gradient characteristics, which have been studied in [36]. Besides, it is discussed the fact that the main result related to the cooperative game approach allows to compute the Shapley value under a distributed structure, i.e., without considering a complete graph connecting the whole set of players. Also, it is shown that this result leads to an additional alternative to compute the Shapley value by using a linear set of equations and using the coalitional rationality axiom (in a centralized manner, or under a distributed structure). As a consequence of the decrement of required communication links to make the computation under distributed structures, there is an increment in the computational cost. However, a comparison between three alternatives to compute the Shapley value, including the computation under a distributed structure, has been added in function of the number of players. This comparison shows that the computation under a distributed structure is faster with respect to the traditional computation of the Shapley value as there is an increment in the number of players.

The remainder of this paper is organized as follows. Section 2 presents the flow-based distribution network and the corresponding control objectives for a resource allocation problem. In addition, it is shown that the considered flowbased distribution network is passive. Section 3 presents the population-games approach used to find the solution given by the Nash equilibrium. Replicator and projection dynamics are also introduced. Afterwards, it is shown that these dynamics satisfy invariance of the simplex set, and that the population games are lossless. Besides, the stability analysis of the closed loop with the flow-based distribution network and the population dynamics is presented. In Section 4, the role of the cooperative-game approach in a particular control problem is shown. This cooperative direction is addressed by finding the solution given by the Shapley value. This section also presents the distributed computation of the Shapley value, including a comparison among the computational burden for the different methodologies used. Also, the partitioning is performed using the Shapley value. Section 5 describes a water distribution network as a case study and the related control objectives. Besides, results for three different sizes of partitions are discussed. Finally, concluding remarks are drawn in Section 6.

\section{Notation:}

Column vectors are denoted by bold style, e.g., x. Matrices are denoted by upper case bold style, e.g., A. Scalar numbers are denoted by non-bold style, e.g., $N$. Sets are denoted by calligraphic upper case, e.g., $\mathcal{T}$. Sub-index refers to the topologies in the system, and super-index refers to partitions, e.g., $\mathcal{V}_{j}^{j}$ refers to a set of a partition $j$ of a topology $i$. It is worth to point out that super-index is not operational, i.e, $N_{i}^{3}$ refers to partition 3 , and not to $N_{i} N_{i} N_{i}$. Real numbers are denoted by $\mathbb{R}$, and all the non-negative real numbers by $\mathbb{R}_{\geq 0}$. Positive integer numbers are denoted by $\mathbb{Z}_{>0}$. The column tile with $N$ unitary entries is denoted by $\mathbb{1}_{N}$, and the cardinality of set is defined by $|\cdot|$, e.g., $|\mathcal{O}|$ is the amount of elements of the set $\mathcal{O}$. Finally, the time dependency in most of the variables is omitted throughout this paper to simplify the notation.

\section{Problem Statement in Distribution Flow-based Networks}

Several engineering problems may be addressed as a flow-based distribution network just by defining general elements such as suppliers and demands, flow capacity, and storage capacity [11]. Consider a specific resource flow-based distribution network, which is composed of three types of elements:

- Storage: element that stores the resource and with both inflows and outflows. Let $\mathcal{V}=\{1, \ldots, N\}$ be the set of $N \in \mathbb{Z}_{>0}$ storage nodes ${ }^{1}$.

- Sink: element that receives the resource from a storage node and with only inflows. Let $\mathcal{B}=\{N+1, \ldots, 2 N\}$ be the set $N \in \mathbb{Z}_{>0}$ of sink nodes.

- Source: element that provides the resource and with only outflows. Let $\mathcal{R}=\{2 N+1\}$ be the singleton set of a source node.

Consider a directed graph denoted by $\tilde{\mathcal{G}}=(\tilde{\mathcal{V}}, \tilde{\mathcal{E}})$, where $\tilde{\mathcal{V}}=\{\mathcal{V} \cup \mathcal{B} \cup \mathcal{R}\}$ is the set of $2 N+1$ nodes representing the storage, sink, and source elements in the flow-based distribution network, and $\tilde{\mathcal{E}} \subset\{(r, \ell): r, \ell \in \tilde{\mathcal{V}}\}$ is the set of

1 The set $\mathcal{V}$ has three different interpretations depending on the context in which it is used, i.e.,

(1) $\mathcal{V}$ is the set of $N$ storage nodes in the distribution flow-based networks context (Section 2).

(2) $\mathcal{V}$ is the set of $N$ strategies in the population-games context (Section 3).

(3) $\mathcal{V}$ is the set of $N$ players in the cooperative-games context (Section 4). 
directed links composed of two ordered pairs of nodes, which represent the resource outflow from $r$, being an inflow to node $\ell$, with $r, \ell \in \tilde{\mathcal{V}}$. On the other hand, consider a bidirectional communication network among the sub-systems, which determines the possible information sharing in order to compute the control inputs. The communication network is represented by an undirected graph denoted by $\mathcal{G}=(\mathcal{V}, \mathcal{E}, \mathbf{A})$, where $\mathcal{E} \subset\{(r, \ell): r, \ell \in \mathcal{V}\}$ is the set of links allowing bidirectional communication between $r, \ell \in \mathcal{V}$, i.e., $(r, \ell)$ and $(\ell, r)$ represent the same link, and $\mathbf{A} \in \mathbb{R}^{N \times N}$ is the adjacency matrix of the graph $\mathcal{G}$. These two graphs are presented in Figure 1 . Notice that the storage elements in the distribution flow-based network presented in Figure 1(a) may be considered as source elements supplying other lower-level storage elements as presented in Figure 2.

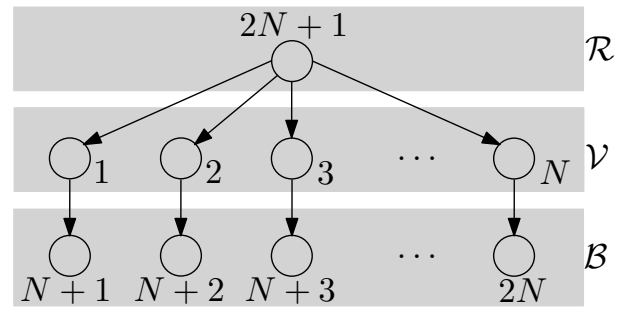

(a)

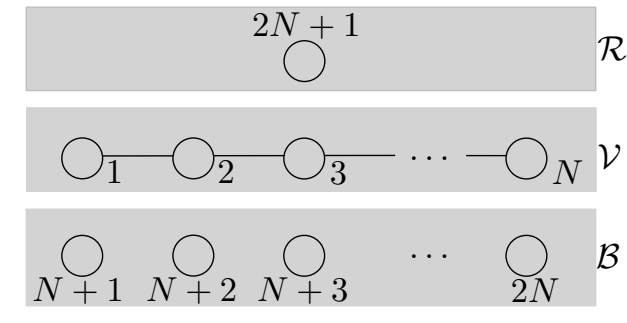

(b)

Fig. 1. Distribution flow-based network. (a) directed graph $\tilde{\mathcal{G}}$ corresponding to the existing network flows. (b) undirected graph example $\mathcal{G}$ corresponding to the communication sharing among sub-systems (associated to storage nodes).

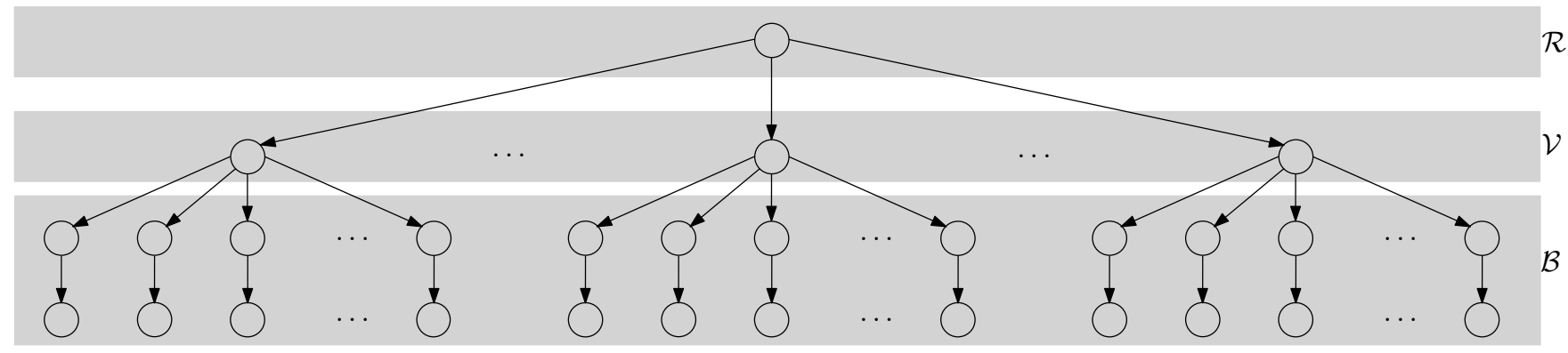

Fig. 2. Tree-shaped topology composed by multiple flow-based distribution networks.

Storage nodes have discharge coefficients given by $K_{\ell}>0$, for all $\ell \in \mathcal{V}$, which determine the outflow resource from the storage nodes.

Furthermore, the inflows of the storage nodes $x_{\ell} \in \mathbb{R}_{>0}$, for all $\ell \in \mathcal{V}$, are manipulated imposing a proportion of resource, i.e., $Q x_{\ell}$, where $0<x_{\ell} \leq 1$, for all $\ell \in \mathcal{V}$, being $Q \in \mathbb{R}_{>0}$ the total resource in the system. Besides, storage nodes have associated a vector of states denoted by $\mathbf{z} \in \mathbb{R}_{>0}^{N}$ determining the amount of resource at each of these nodes. The storage nodes show the following dynamics:

$$
\dot{z}_{\ell}=Q x_{\ell}-K_{\ell} z_{\ell}, \text { for all } \ell \in \mathcal{V}
$$

where the equilibrium duple $\left(x_{\ell}^{*}, z_{\ell}^{*}\right)$ implies that a non-null steady state has been achieved for the stored resource, i.e., $z_{\ell}^{*}>0$ since $x_{\ell}^{*}>0$. In the aforementioned flow-based network, the resource $Q$ is distributed throughout the storage nodes, which can be seen as sub-systems within the network. For this distribution system, there are two objectives. First, it is desired to make an evenhanded distribution of a resource throughout different sub-systems, i.e.,

$$
\underset{\mathbf{x}}{\operatorname{minimize}}\left(z_{\ell}-\frac{1}{N} \sum_{\ell=1}^{N} z_{\ell}\right), \text { for all } \ell \in \mathcal{V} .
$$

As a second objective, it is desired to determine the appropriate distribution of costs for the sub-systems in function of their contribution to the first control objective, which is attained by using the available communication channels to 
coordinate the distribution of the resource. The communication cost in an time interval $\left[t_{0}, t_{f}\right]$ associated to the use of $\mathcal{G}=(\mathcal{V}, \mathcal{E}, \mathbf{A})$ is given by $\varphi_{\text {links }}=\frac{1}{2} \int_{t_{0}}^{t_{f}} \mathbb{1}_{N}^{\top} \mathbf{A} \mathbb{1}_{N} d t$. As a second objective, the fair cost $L_{\ell}$ that the $\ell^{\text {th }}$ sub-system should pay for using the communication network must be found. It should be satisfied that $\sum_{\ell \in \mathcal{V}} L_{\ell}=\varphi_{\text {links }}$.

Finally, assume that the communication graph can have $T$ different topologies from the set $\mathcal{T}=\{1, \ldots, T\}$. Moreover, each topology $i \in \mathcal{T}$ has $P_{i}$ different partitions from the set $\mathcal{P}_{i}=\left\{1, \ldots, P_{i}\right\}$, where each partition $j \in \mathcal{P}_{i}$ of the topology $i \in \mathcal{T}$ is a complete undirected graph denoted by $\mathcal{G}_{i}^{j}=\left(\mathcal{V}_{i}^{j}, \mathcal{E}_{i}^{j}, \cdot\right)$, where $\mathcal{V}_{i}^{j}$ is the set of $N_{i}^{j}$ storage nodes within the corresponding partition, and $\mathcal{E}_{i}^{j}=\left\{(r, \ell): r, \ell \in \mathcal{V}_{i}^{j}\right\}$ is the set of links representing the full-information within each partition. Besides, each partition $j \in \mathcal{P}_{i}$ of the topology $i \in \mathcal{T}$ represents a sub-system denoted by $\boldsymbol{\Sigma}_{i}^{j}$, which has a disjoint controller denoted by $\boldsymbol{\Gamma}_{i}^{j}$ as presented in Figure 3 .

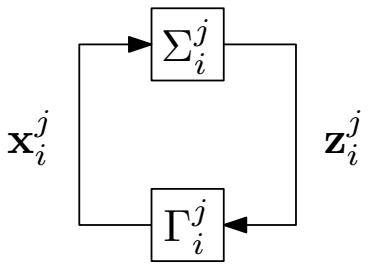

Fig. 3. Closed loop for the sub-system corresponding to partition $j \in \mathcal{P}_{i}$ of the topology $i \in \mathcal{T}$.

The two different control objectives are achieved by using a game-theoretical approach in a distributed manner. The first control objective associated to an evenhanded distribution of the resource is reached with a non-cooperative approach. In contrast, the determination of the fair distribution of costs for all the sub-systems is made with a cooperative game approach. Furthermore, suppose that the topology of the communication graph can be reconfigured conveniently to achieve the control objectives every time $\tau>0$ (this fact is discussed in Section 3).

A feature of the flow-based distribution network is its passivity property. Lemma 1 presents this property as in [1][8].

Lemma 1 The storage model of the flow-based distribution network $\boldsymbol{\Sigma}_{i}^{j}$ in Figure 3 is passive defining its inputs as the error $e_{z_{\ell}}=z_{\ell}-z_{\ell}^{*}$, and its outputs as the error $e_{x_{\ell}}=x_{\ell}-x_{\ell}^{*}$, for all $\ell \in \mathcal{V}_{i}^{j}$, and for all partitions $j \in \mathcal{P}_{i}$ of the topology $i \in \mathcal{T}$.

Proof: The proof is presented in the Appendix.

\section{Population Games}

Consider a population composed of a finite and large number of rational agents that make the decisions to select among a set of possible strategies $\mathcal{V}=\{1, \ldots, N\}$ (each strategy associated to a storage node, see Section 2). Agents change the strategy to improve their utilities or benefits.

Within the population there are $T \in \mathbb{Z}_{>0}$ possible different topologies given by a graph that determine how the population structure is configured, i.e., how the agents can interact among them. The set of the population topologies is denoted by $\mathcal{T}=\{1, \ldots, T\}$. Each topology $i \in \mathcal{T}$ is given by a non-complete graph denoted by $\mathcal{G}_{i}=\left(\mathcal{V}, \mathcal{E}_{i}, \mathbf{A}_{i}\right)$, where $\mathcal{V}$ is the set of $N$ nodes representing the strategies, the set of links that represent the possible interaction among agents selecting the corresponding strategies is denoted by $\mathcal{E}_{i}$, and $\mathbf{A}_{i}$ is the adjacency matrix of the corresponding topology.

Each topology $i \in \mathcal{T}$ has $P_{i} \in \mathbb{Z}_{>0}$ disjoint partitions. The set of partitions of the population topology $i \in \mathcal{T}$ is given by $\mathcal{P}_{i}=\left\{1, \ldots, P_{i}\right\}$. The partition $j \in \mathcal{P}_{i}$ of the topology $i \in \mathcal{T}$ is denoted by a complete graph $\mathcal{G}_{i}^{j}=\left(\mathcal{V}_{i}^{j}, \mathcal{E}_{i}^{j}, \cdot\right)$, where $\mathcal{V}_{i}^{j}$ is the set of $N_{i}^{j}<N$ nodes representing the set of strategies within the corresponding partition $\mathcal{V}_{i}^{j} \subset \mathcal{V}$, and $\mathcal{E}_{i}^{j}$ is the set of $N_{i}^{j}\left(N_{i}^{j}-1\right) / 2$ links representing the full-information sharing and interaction within each partition. Furthermore, it must be satisfied that all the partitions form the entire topology, i.e., $\bigcup_{j \in \mathcal{P}_{i}} \mathcal{G}_{i}^{j}=\mathcal{G}_{i}$, for all $i \in \mathcal{T}$.

In the population, the scalar $x_{\ell} \in \mathbb{R}_{\geq 0}$ is the proportion of agents selecting the strategy $\ell \in \mathcal{V}$. The vector $\mathbf{x} \in \mathbb{R}_{>0}^{N}$ is a population state or a strategic distribution composed of all the proportion of agents selecting the available strategies. 
The set of all the possible population states is given by a simplex denoted by $\Delta=\left\{\mathbf{x} \in \mathbb{R}_{\geq 0}^{N}: \mathbf{x}^{\top} \mathbb{1}_{N}=1\right\}$. Similarly, $x_{i, \ell}^{j} \in \mathbb{R}_{\geq 0}$ is the proportion of agents selecting the strategy $\ell \in \mathcal{V}_{i}^{j}$ available in the partition $j \in \mathcal{P}_{i}$ of the topology $i \in \mathcal{T}$. The vector $\mathbf{x}_{i}^{j} \in \mathbb{R}_{\geq 0}^{N_{i}^{j}}$ is the strategic distribution of agents in the partition $j \in \mathcal{P}_{i}$ of the topology $i \in \mathcal{T}$. Finally, let $m_{i}^{j}$ be the total mass in the partition $j \in \mathcal{P}_{i}$ of the topology $i \in \mathcal{T}$ given by $m_{i}^{j}=\mathbf{x}_{i}^{j} \mathbb{1}_{N_{i}^{j}}$. Since partitions are disjoint, then $\sum_{j \in \mathcal{P}_{i}} m_{i}^{j}=1$.

The payoff that agents receive for selecting a particular strategy is given by a fitness function $f_{\ell}: \Delta \mapsto \mathbb{R}$, for the associated strategy $\ell \in \mathcal{V}$. The vector of fitness functions in the population is denoted by $\mathbf{f}$ and is composed of all the fitness functions $f_{\ell}(\mathbf{x}), \ell \in \mathcal{V}$. Similarly, the vector of fitness functions of the partition $j \in \mathcal{P}_{i}$ of the topology $i \in \mathcal{T}$ is denoted by $\mathbf{f}_{i}^{j}$, which is composed of all the fitness functions $f_{\ell}(\mathbf{x}), \ell \in \mathcal{V}_{i}^{j}$. The average fitness function in the population is given by $\bar{f}=\mathbf{x}^{\top} \mathbf{f}$. The average fitness for each partition of each topology is given by $\bar{f}_{i}^{j}=\left(\mathbf{x}_{i}^{j^{\top}} \mathbf{f}_{i}^{j}\right) / m_{i}^{j}, j \in \mathcal{P}_{i}, i \in \mathcal{T}$, and the average fitness vector for the partition $j \in \mathcal{P}_{i}$ of the topology $i \in \mathcal{T}$ is $\overline{\mathbf{f}}_{i}^{j}=\mathbb{1}_{N_{i}^{j}} \bar{f}_{i}^{j}$.

The framework proposed in this paper is given by stable games, which establish conditions over the fitness functions for control design. The condition of stable games stated in Definition 1 allows proving asymptotic convergence to the solution in the non-cooperative game approach addressed by population games as presented in Section 3.2.

Definition 1 (Adapted from [12]) $\mathbf{f}$ is a stable game if the Jacobian matrix D) is negative semi-definite with respect to the tangent space denoted by $\Delta_{T}=\left\{\mathbf{z} \in \mathbb{R}^{N}: \mathbf{z}^{\top} \mathbb{1}_{N}=0\right\}$, i.e., $\mathbf{z}^{\top} D \mathbf{f} \mathbf{z} \leq 0$, for all $\mathbf{z} \in \Delta_{T}, \mathbf{x} \in \Delta$. Alternatively, $(\mathbf{w}-\mathbf{x})^{\top}(\mathbf{f}(\mathbf{w})-\mathbf{f}(\mathbf{x})) \leq 0$, for all $\mathbf{x}, \mathbf{w} \in \Delta$.

In this paper, both replicator and projection dynamics are used [35]. These two population dynamics are especially appealing since they have gradient properties that are discussed in [36].

\subsection{Replicator and Projection Dynamics}

For a fixed topology, there is a replicator dynamics system for each partition. For a topology $i \in \mathcal{T}$ and a partition $j \in \mathcal{P}_{i}$, the replicator dynamics introduced in [38] are given by

$$
\dot{\mathbf{x}}_{i}^{j}=\operatorname{diag}\left(\mathbf{x}_{i}^{j}\right)\left(\mathbf{f}_{i}^{j}-\overline{\mathbf{f}}_{i}^{j}\right)
$$

and the projection dynamics introduced in [23] are given by

$$
\dot{\mathbf{x}}_{i}^{j}=\mathbf{f}_{i}^{j}-\frac{1}{N_{i}^{j}} \mathbb{1}_{N_{i}^{j}} \mathbf{f}_{i}^{j^{\top}} \mathbb{1}_{N_{i}^{j}} .
$$

Then, the system changes among topologies ${ }^{2}$ in order to use, at each iteration, a limited number of communication links. The equilibrium of interest in (2) for this work is the non-pure Nash equilibrium given by the condition $\mathbf{f}_{i}^{j *}=\overline{\mathbf{f}}_{i}^{j *}$, for all $j \in \mathcal{P}_{i}, i \in \mathcal{T}$. This is equivalent to $\mathbf{f}_{i}^{j *} \in \operatorname{span}\left\{\mathbb{1}_{N_{i}^{j}}\right\}$, for all $i \in \mathcal{T}$ and $j \in \mathcal{P}_{i}$. Notice that the dynamics (2) have an equilibrium point $\mathbf{x}_{i}^{j *}=0$, which implies the extinction of the agents. Consequently, it is assumed that, under the replicator dynamics (2), there is not extinction of proportion of agents. Regarding the equilibrium for (3), it is achieved when all the fitness functions get the same value, i.e., $\mathbf{f}_{i}^{j *} \in \operatorname{span}\left\{\mathbb{1}_{N_{i}^{j}}\right\}$, for all $i \in \mathcal{T}$ and $j \in \mathcal{P}_{i}$. Due to the fact that each topology is a non-connected graph, this equilibrium is achieved at each partition. Moreover, since topologies and partitions are varying over time, it is necessary to identify the equilibrium for all topologies and for all partitions, i.e., $f_{k}\left(\mathbf{x}^{*}\right)=f_{\ell}\left(\mathbf{x}^{*}\right)$, for all $k, \ell \in \mathcal{V}$.

Remark 1 Suppose that $\mathbf{f}$ is a stable game, then $\left(\mathbf{x}-\mathbf{x}^{*}\right)^{\top}\left(\mathbf{f}(\mathbf{x})-\mathbf{f}\left(\mathbf{x}^{*}\right)\right) \leq 0$ according to Definition 1. Moreover, due to the fact that $\mathbf{f}\left(\mathbf{x}^{*}\right) \in \operatorname{span}\left\{\mathbb{1}_{N}\right\}$, and that $\mathbf{x} \in \Delta, \mathbf{x}^{*} \in \Delta$, then $\left(\mathbf{x}-\mathbf{x}^{*}\right)^{\top} \mathbf{f}\left(\mathbf{x}^{*}\right)=0$. This fact leads to $\left(\mathbf{x}-\mathbf{x}^{*}\right)^{\top} \mathbf{f}(\mathbf{x}) \leq 0$.

$\overline{2}$ These topologies are determined by a partitioning performed by using a cooperative game approach, which is later discussed in this paper. 
Once some population dynamics have been introduced, i.e., replicator and projection dynamics, their properties such the invariance of the simplex set under these dynamics and the convergence to the equilibrium point are presented next.

\subsection{Population Dynamics Properties}

The first property of both replicator and projection dynamics is the invariance of the simplex set $\Delta$, which is analyzed in Proposition 1.

Proposition 1 Let $\mathbf{x}(0)$ be the initial condition of (2) or (3). If $\mathbf{x}(0) \in \Delta$, then $\mathbf{x} \in \Delta$, for all $t \geq 0$, i.e., the simplex $\Delta$ is an invariant set under replicator dynamics (2) or projection dynamics (3), for any partition topology.

Proof: The proof is presented in the Appendix.

Now, the passivity property of both the replicator and the projection dynamics is analyzed in Lemma 2.

Lemma 2 In Figure 3, $\boldsymbol{\Gamma}_{i}^{j}$ can be given by either the replicator dynamics (2) or the projection dynamics (3). For any of these dynamics, $\boldsymbol{\Gamma}_{i}^{j}$ is lossless defining its inputs as $\mathbf{f}_{i}^{j}$, and its outputs as $\mathbf{x}_{i}^{j}-\mathbf{x}_{i}^{j *}$, for all partitions $j \in \mathcal{P}_{i}$ of the topology $i \in \mathcal{T}$.

Proof: The proof is presented in the Appendix.

Remark 2 According to Remark 1 and taking into account the derivative of the storage functions (also Lyapunov functions) $\dot{L}_{2} \leq 0$, and $\dot{L}_{3} \leq 0$, there exists a time $\tau>0$ such that $\left\|x_{\ell}(t)-x_{\ell}^{*}\right\| \geq\left\|x_{\ell}(t+\tau)-x_{\ell}^{*}\right\|$, for all $\ell \in \mathcal{V}$. $\diamond$

Once the passivity features of the considered flow-based distribution network and both the replicator and projection dynamics have been presented, it is shown that the equilibrium point of the closed-loop system in Figure 3 is stable as stated in Proposition 2.

Proposition 2 The equilibrium pair $\left(\mathbf{z}_{i}^{j *}, \mathbf{x}_{i}^{j *}\right)$, for all partitions $j \in \mathcal{P}_{i}$ in the topology $i \in \mathcal{T}$ (i.e., the equilibrium $\mathbf{z}_{i}^{j *}$ of the sub-system $\boldsymbol{\Sigma}_{i}^{j}$, and the equilibrium $\mathbf{x}_{i}^{j *}$ of the system $\boldsymbol{\Gamma}_{i}^{j}$, for the closed loop presented in Figure 3) is stable under the replicator and projection dynamics selecting $\mathbf{f}_{i}^{j}=\mathbf{z}_{\max , i}^{j}-\mathbf{z}_{i}^{j}$, where $\mathbf{z}_{\max , i}^{j}$ is the constant maximum capacity of the storage nodes belonging to $\mathcal{V}_{i}^{j}$.

Proof: The proof is presented in the Appendix.

Notice that the population-games-based controller in Figure 3 is of data-driven nature since it is designed without requiring the model of the system.

\section{Coalitional-Game Role and Partitioning Criterion}

Consider a cooperative game with transferable utility defined as a pair $(\mathcal{V}, V)$, where $\mathcal{V}=\{1, . ., N\}$ is the set of players (each player associated to a storage node, see Section 2), and $V$ is the characteristic function. From the cooperative-game viewpoint for each topology $i \in \mathcal{T}$, each node $\ell \in \mathcal{V}$ is a player and each partition $j \in \mathcal{P}_{i}$ represents a coalition of players.

\subsection{Computation of the Shapley Value}

The characteristic function $V$ assigns a real value to each of the $2^{\mathcal{V}}$ coalitions and returns a real value. Formally, the characteristic function is a mapping $V: 2^{\mathcal{V}} \mapsto \mathbb{R}$. For each coalition, $\mathcal{O} \subseteq \mathcal{V}, V(\mathcal{O})$ is the value that players can share among themselves. Additionally, for the empty coalition, $V(\emptyset) \triangleq 0$. 
Prior to defining the characteristic function, costs associated to each coalition are defined as

$$
C(\mathcal{O})=\frac{1}{|\mathcal{O}|} \sum_{\ell \in \mathcal{O}} C_{\ell}
$$

where $C_{\ell}$ is the individual cost of player belonging to the coalition $\ell \in \mathcal{O}$. For the considered flow-based distribution networks, costs are defined to be $C_{\ell}=z_{\max , \ell}-z_{\ell}$, for all $\ell \in \mathcal{V}$, where $z_{\max , \ell}$ is the maximum capacity of the storage node, and $z_{\ell}$ is the current state of the corresponding storage node. This individual value represents a cost that a player would have to assume in case that it does not cooperate with anyone. Notice that the error $C_{\ell}$ is an appropriate selection for the costs since a player $\ell$ with null error does not have incentives to cooperate with others due to the fact it has achieved the first control objective, and cooperation would imply to increment the error. In contrast, a player with a big error $C_{\ell}$ has incentives to cooperate in order to minimize its error and should assume costs for it. In addition, if two players have identical errors $C_{\ell}$, then it is reasonable that both assume the same costs to cooperate each other. In other words, it is reasonable that those players with bigger errors incur in higher costs to achieve the first control objective.

Furthermore, the individual cost allows the player to determine its incentives to establish a coalition with another player, i.e., the player would have incentives to cooperate with others as long as the cooperation implies a reduction of its costs. Besides, these individual costs allow computing the contribution of a player to a coalition, i.e., how the cost is reduced as the player collaborates with an existing coalition. Afterwards, the specific considered characteristic function is given by the difference between the sum of individual costs and the cost of the corresponding coalition. Then, the difference between the costs when each player operates by itself and the costs when all these players collaborate with each other can be used to define the cost function as

$$
V(\mathcal{O})=\sum_{\ell \in \mathcal{O}} C_{\ell}-C(\mathcal{O}) .
$$

A solution of the cooperative game is an allocation rule that provides each player with a payoff according to its contribution. Let $\mathbf{y} \in \mathbb{R}^{N}$ be the payoff vector given by $\mathbf{y}=\left[\begin{array}{lll}y_{1} & \cdots & y_{N}\end{array}\right]^{\top}$. Some desirable properties for the distribution of the $V(\mathcal{O})$ among the players are:

(1) Efficiency: $\sum_{\ell \in \mathcal{O}} y_{\ell} \leq V(\mathcal{V})$,

(2) Coalitional rationality: $\sum_{\ell \in \mathcal{O}} y_{\ell}=V(\mathcal{O})$ for all coalitions $\mathcal{O} \subseteq \mathcal{V}$,

(3) Individual rationality: $y_{\ell} \geq V(\{\ell\})$, for all $\ell \in \mathcal{V}$.

A payoff rule that satisfies the mentioned desired requirements is the Shapley value (or Shapley power index) [28][37], which is given by

$$
\Phi(\ell)=\sum_{\mathcal{O} \subseteq \mathcal{V} \backslash\{\ell\}} \Psi(\mathcal{O})(V(\mathcal{O} \cup\{\ell\})-V(\mathcal{O})),
$$

where $\Psi(\mathcal{O})=(|\mathcal{O}| !(N-|\mathcal{O}|-1) !) /(N !)$. Notice that the sum considers all the possible coalitions where player $\ell \in \mathcal{V}$ can be added. Its computation requires full information from all the players and coalitions of the cooperative game, resulting in high computational burden. In particular, the combinatorial explosion when having a high number of players is a common issue in this context.

Once the characteristic function has been defined as in (5), a mathematical relationship between the Shapley values can be determined to mitigate the high computational cost enhancing the possibilities to use a distributed structure. This result is presented in Proposition 3.

Proposition 3 Let (5) be the characteristic function of a cooperative game with the set of players $\mathcal{V}=\{1, \ldots, N\}$. Let $C_{\ell}$ be the cost associated to each player $\ell \in \mathcal{V}$, and (4) be the costs associated to each coalition $\mathcal{O} \subseteq \mathcal{V}$. The Shapley value $\Phi(\ell)$ for any player $\ell \in \mathcal{V}$ is computed as follows:

$$
\Phi(\ell)=\frac{1}{N}\left(V(\mathcal{V})-\Theta\left(\sum_{r \in \mathcal{V} \backslash\{\ell\}} C_{r}-(N-1) C_{\ell}\right)\right),
$$


where $\Theta>0$ is a constant for the cooperative game whose value only depends on the number of players $N$, i.e.,

$$
\Theta=\sum_{s=1}^{N-2}\left\{\left(\frac{(N-2) !}{s !(N-2-s) !}\right)\left(\frac{s}{s+1}\right)\left(\frac{s !(N-s-1) !+(s+1) !(N-s-2) !}{N !}\right)\right\} .
$$

Besides, there is a relationship between Shapley values given by $\Phi(r)=\Phi(\ell)+\left(C_{r}-C_{\ell}\right) \Theta$, for all $r, \ell \in \mathcal{V}$.

Proof: First, it is proven the relationship between the Shapley values of different players with the constant $\Theta$ given by $\Phi(r)=\Phi(\ell)+\left(C_{r}-C_{\ell}\right) \Theta$, for all $r, \ell \in \mathcal{V}$. The Shapley value $\Phi(\ell)$ of the player $\ell \in \mathcal{V}$ in (6) may be re-written by expressing the set of coalitions to which the player $\ell \in \mathcal{V}$ can be added, in terms of a second player $r \in \mathcal{V}$, as follows:

$$
\Phi(\ell)=\sum_{\mathcal{O} \subseteq \mathcal{V} \backslash\{\ell, r\}} \Psi(\mathcal{O})(V(\mathcal{O} \cup\{\ell\})-V(\mathcal{O}))+\sum_{\mathcal{O} \subseteq \mathcal{V} \backslash\{\ell, r\}} \Psi(\mathcal{O} \cup\{r\})(V(\mathcal{O} \cup\{r\} \cup\{\ell\})-V(\mathcal{O} \cup\{r\})) .
$$

Similarly, the Shapley value $\Phi(r)$ of the player $r \in \mathcal{V}$ may be written in terms of player $\ell \in \mathcal{V}$ as follows:

$$
\Phi(r)=\sum_{\mathcal{O} \subseteq \mathcal{V} \backslash\{\ell, r\}} \Psi(\mathcal{O})(V(\mathcal{O} \cup\{r\})-V(\mathcal{O}))+\sum_{\mathcal{O} \subseteq \mathcal{V} \backslash\{\ell, r\}} \Psi(\mathcal{O} \cup\{\ell\})(V(\mathcal{O} \cup\{r\} \cup\{\ell\})-V(\mathcal{O} \cup\{\ell\})) .
$$

Now, it is found the difference between the Shapley values $\Phi(r)$ and $\Phi(\ell)$, denoted by $\tilde{\Phi}(r, \ell)=\Phi(r)-\Phi(\ell)$. Here it is taken into account that $\Psi(\mathcal{O} \cup\{r\})=\Psi(\mathcal{O} \cup\{\ell\})$. Hence

$$
\tilde{\Phi}(r, \ell)=\sum_{\mathcal{O} \subseteq \mathcal{V} \backslash\{\ell, r\}} \Psi(\mathcal{O})\{V(\mathcal{O} \cup\{r\})-V(\mathcal{O} \cup\{\ell\})\}+\sum_{\mathcal{O} \subseteq \mathcal{V} \backslash\{\ell, r\}} \Psi(\mathcal{O} \cup\{r\})\{V(\mathcal{O} \cup\{r\})-V(\mathcal{O} \cup\{\ell\})\} .
$$

Replacing (5) and (4) in (8), it is obtained

$$
\tilde{\Phi}(r, \ell)=\sum_{\mathcal{O} \subseteq \mathcal{V} \backslash\{\ell, r\}} \Psi(\mathcal{O})\left\{\left(1-\frac{1}{|\mathcal{O}|+1}\right)\left(C_{r}-C_{\ell}\right)\right\}+\sum_{\mathcal{O} \subseteq \mathcal{V} \backslash\{\ell, r\}} \Psi(\mathcal{O} \cup\{r\})\left\{\left(1-\frac{1}{|\mathcal{O}|+1}\right)\left(C_{r}-C_{\ell}\right)\right\} .
$$

Briefly, the difference between the Shapley values $\tilde{\Phi}(r, \ell)=\Phi(r)-\Phi(\ell)$ is given by

$$
\tilde{\Phi}(r, \ell)=\left(C_{r}-C_{\ell}\right) \underbrace{\sum_{\mathcal{O} \subseteq \mathcal{V} \backslash\{\ell, r\}} \overbrace{(\Psi(\mathcal{O})+\Psi(\mathcal{O} \cup\{r\}))}^{\theta_{1}} \overbrace{\left(\frac{|\mathcal{O}|}{|\mathcal{O}|+1}\right)}^{\theta_{2}}}_{\Theta} .
$$

Notice that the constant value $\Theta$ can be re-written as

$$
\Theta=\sum_{s=1}^{N-2}\{\overbrace{\left(\frac{(N-2) !}{s !(N-2-s) !}\right)}^{\theta_{3}} \overbrace{\left(\frac{s}{s+1}\right)}^{\theta_{2}} \underbrace{\left(\frac{s !(N-s-1) !+(s+1) !(N-s-2) !}{N !}\right)}_{\theta_{1}}\},
$$

where $\theta_{3}$ represents the amount of coalitions that can be formed in the cooperative game with $s$ players, i.e., $|\mathcal{O}|=s$. Finally, $\Phi(r)=\Phi(\ell)+\left(C_{r}-C_{\ell}\right) \Theta$, obtaining the desired relationship from which it follows:

$$
\sum_{r \in \mathcal{V} \backslash\{\ell\}} \Phi(r)=(N-1) \Phi(\ell)+\Theta \sum_{r \in \mathcal{V} \backslash\{\ell\}} C_{r}-\Theta(N-1) C_{\ell},
$$


adding $\Phi(\ell)$ at both sides yields $\sum_{r \in \mathcal{V}} \Phi(r)=N \Phi(\ell)+\Theta\left(\sum_{r \in \mathcal{V}} C_{r}-(N-1) C_{\ell}\right)$, and since $V(\mathcal{V})=\sum_{r \in \mathcal{V}} \Phi(r)$, then

$$
\Phi(\ell)=\frac{1}{N}\left(V(\mathcal{V})-\Theta\left(\sum_{r \in \mathcal{V} \backslash\{\ell\}} C_{r}-(N-1) C_{\ell}\right)\right),
$$

completing the proof.

Remark 3 If $C_{r}>C_{\ell}$, then $\Phi(r)>\Phi(\ell)$, for all $r, \ell \in \mathcal{V}$. Suppose that $C_{r}>C_{\ell}$, and since $\Theta>0$, then $\Phi(r)=\Phi(\ell)+\left(C_{r}-C_{\ell}\right) \Theta>\Phi(\ell)$.

\subsection{Computation of the Shapley Value under a Distributed Structure}

The reduction of computation time according to the result obtained in Proposition 3 allows investing extra computational effort to gather all the needed information to compute the Shapley value under a distributed structure. In order to make the distributed Shapley computation, let one player be a pivot player in charge of collecting all the required information from all the other players known as supply players.

Remark 4 Even though the computation of the Shapley value is performed by one player, the required structure is a non-complete graph. In this regard, the computation of the Shapley value is made under a distributed structure. $\diamond$

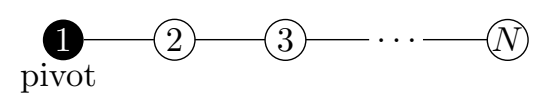

Fig. 4. Path graph for the distributed Shapley computation. Without loss of generality, the pivot player is considered to be player 1.

Consider a connected non-complete graph for the distributed computation of the Shapley value denoted by $\mathcal{G}=(\mathcal{V}, \mathcal{E})$, where $\mathcal{V}$ is the set of nodes representing the players within the cooperative game. Moreover, let $p \in \mathcal{V}$ denote the pivot player, and $\mathcal{V} \backslash\{p\}$ is the set of supply players. $\mathcal{E}$ is the set of links that represent possible communication among the players. Moreover, let $\mathcal{N}_{\ell}$ be the set of neighbors of player $\ell \in \mathcal{V}$, i.e., $\mathcal{N}_{\ell}=\{r:(\ell, r) \in \mathcal{E}\}$. Figure 4 shows the case for a path graph where the first player is the pivot player, i.e., $p=1 \in \mathcal{V}$. Notice that the required information to compute the Shapley value is given by all the individual costs of all the supply players according to (4) (since the pivot player knows its own individual cost), and the total number of players. This information is required by the pivot player who is in charge of the Shapley value computation in order to perform the partitioning (which is used for establishing the proper topologies introduced in Section 3) and also assign the fair distribution of costs associated to the communication links. It is necessary that the pivot player obtains the information in a distributed way subject to the communication topology given by the graph $\mathcal{G}$.

The distributed algorithm is inspired by the work presented in [9], and it is divided into two different tasks, i.e., the distributed computation of the number of players $N$, and the distributed computation of all the individual costs for all the supply players $C_{\ell}$, for all $\ell \in \mathcal{V} \backslash\{p\}$. Each stage of the algorithm is presented next.

\subsubsection{Computation of the Number of Players}

Consider an auxiliary variable for the pivot player $\xi_{p}$, and for each supply player $\xi_{\ell}$, where $\ell \in \mathcal{V} \backslash\{p\}$. The initial conditions of these auxiliary variables are given by $\xi_{p}(0)=1$, and $\xi_{\ell}(0)=0$, for all $\ell \in \mathcal{V} \backslash\{p\}$. The following consensus algorithm can be implemented taking advantage of the relationship between the initial conditions and the stationary point as $[27][5]$

$$
\dot{\xi}_{\ell}=\sum_{r \in \mathcal{N}_{\ell}}\left(\xi_{r}-\xi_{\ell}\right), \quad \text { for all } \quad \ell \in \mathcal{V}
$$

Since the communication graph $\mathcal{G}$ among players is connected, according to [27] the stationary value of (9) is $\xi_{\ell}^{*}=\left(\sum_{r \in \mathcal{V}} \xi_{r}(0)\right) / N$, for all $\ell \in \mathcal{V}$. Consequently, the stationary value is given by $\xi_{\ell}^{*}=N^{-1}$, for all $\ell \in \mathcal{V}$. This fact shows that the pivot player can get information about the total number of players $N$ in a distributed way. 


\subsubsection{Computation of the Individual Costs}

For simplicity, it is assumed that the pivot player is the player $p=1 \in \mathcal{V}$ (see, e.g., Figure 4 ). Since there are $N-1$ values for individual costs that should be sent to the pivot player, then there is a turn value denoted by $\pi \in \mathbb{Z}_{>0}$. The token assigns a flag for each player to distribute its individual cost. For the case with a pivot player $p=1 \in \mathcal{V}$, the turn value $\pi$ should vary from 2 to $N$ in order to cover the total number of players in the cooperative game. After determining the possible values for the turn variable $\pi$, i.e., $\pi=2, \ldots, N$, this variable is initialized for the first supply player, i.e., $\pi=2$. Once the pivot player determines the individual cost corresponding to this supply player, the turn value is increased, i.e., $\pi=\pi+1$ in order to allow the next supply player distribute its individual cost. This process is repeated until the last player distributes its cost to the pivot player, i.e., until $\pi=N$.

Consider the auxiliary variables $\psi_{\ell}$, for all $\ell \in \mathcal{V}$. The initial conditions of these auxiliary variables are given by $\psi_{\pi}=C_{\pi}$ from (4), and $\psi_{\ell}=0$, for all $\ell \in \mathcal{V} \backslash\{\pi\}$. Then, the consensus algorithm [27] is implemented, i.e,

$$
\dot{\psi}_{\ell}=\sum_{r \in \mathcal{N}_{\ell}}\left(\psi_{r}-\psi_{\ell}\right), \quad \text { for all } \ell \in \mathcal{V}
$$

The stationary value is given by $\psi_{\ell}^{*}=\frac{1}{N} \sum_{r \in \mathcal{V}} \psi_{r}(0)$. For the selected initial conditions, $\psi_{\ell}^{*}=N^{-1} C_{\pi}$. Furthermore, since the number of players is already known from the procedure presented in Subsection 4.2.1, then

$$
\psi_{\ell}^{*}=C_{\pi} \xi_{\ell}^{*}, \quad \text { for all } \pi \in \mathcal{V} \backslash\{p\}
$$

It is concluded that, finding the number of players and finding each individual cost by assigning turns to the supply players, it is possible to compute the Shapley value in a distributed way.

Remark 5 Notice that it is necessary to compute $N$ distributed consensus algorithms, one algorithm for the determination of the number of players, and $N-1$ algorithms to distribute the individual costs of supply players to the pivot player. This fact implies an extra computational burden. However, it is shown that the distributed computation of the Shapley value using distributed consensus and Proposition 3 is lower than the computational burden of the classical approach (6) as the total number of players is increased.

In order to verify the difference between the computational burden of computing the Shapley value with (6), and by using the relation in Proposition 3 with the constant value $\Theta$, different Shapley values for several amount of players have been computed.

Table 1

\begin{tabular}{|c|c|c|c|}
\hline $\begin{array}{c}\text { Total Number } \\
\text { of players } \\
N\end{array}$ & $\begin{array}{c}\text { Centralized approach } \\
\text { by using }(6) \\
\text { time }[\mathrm{s}]\end{array}$ & $\begin{array}{c}\text { Centralized approach } \\
\text { by using }(7) \\
\text { time }[\mathrm{ms}]\end{array}$ & $\begin{array}{c}\text { Distributed approach } \\
\text { by using }(7) \\
\text { time }[\mathrm{s}]\end{array}$ \\
\hline 3 & 0.4232 & 0.09 & 2.7899 \\
\hline 4 & 0.8020 & 0.12 & 3.6002 \\
\hline 6 & 1.2907 & 0.18 & 5.4838 \\
\hline 8 & 2.3421 & 0.24 & 7.2707 \\
\hline 10 & 5.9998 & 0.30 & 9.0004 \\
\hline 12 & 25.6436 & 0.36 & 10.8073 \\
\hline 14 & 110.6065 & 0.42 & 12.6004 \\
\hline 16 & 1944.7919 & 0.48 & 14.4005 \\
\hline 18 & 53938.9433 & 0.54 & 16.2210 \\
\hline 100 & - & 3 & 90.0030 \\
\hline
\end{tabular}

Comparison of computational burden for computing the Shapley value with different number of players.

The comparison among the computation of the Shapley value in a distributed way using Proposition 3 , in a centralized way using Proposition 3, and in a centralized way using (6), is presented in Table 1. 


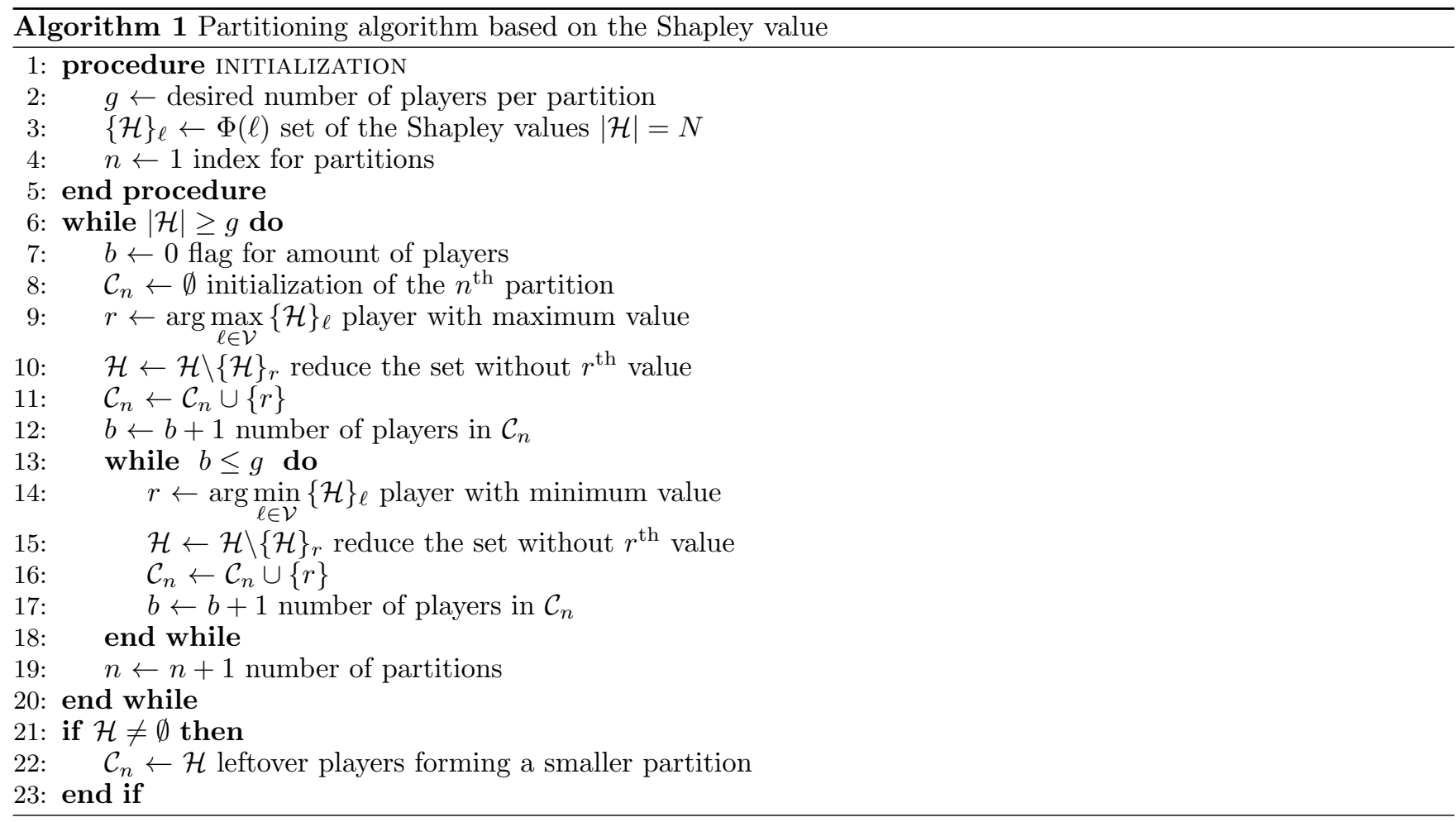

\subsection{Partitioning Procedure}

In order to perform the partitioning of the system, first it is established a time $\tau$ that satisfies Remark 2, which defines when the proper topology is evaluated ${ }^{3}$. Every time $\tau$, the Shapley value $\Phi(\ell)$ of all the players $\ell \in \mathcal{V}$ is computed by using the low-computational-cost operation with the factor $\Theta$. A second necessary parameter is the size of the desired partitions, denoted by $g \in \mathbb{Z}_{>0}$, i.e., make partitions with a number $g<N$ of players.

In the partitioning procedure, it is desired to gather the player with the highest Shapley value with the $g-1$ players with the lowest Shapley values. In this sense, it is possible to make a cooperation in which the best players share their benefits with those in worse situation. Details of this partitioning process at every time $\tau$ are presented in Algorithm 1. It is worth to highlight that this partitioning criterion might be different depending on the control objectives and the system dynamical behavior. In this paper, the partitioning is performed in function of the Shapley value by grouping players with the highest power index with those with lowest power index. This procedure allows players to unify their power index and therefore to achieve an evenhanded distribution of resource.

Lemma 3 The stable closed-loop system presented in Figure 3, changing the partitions every $\tau$ by using the Shapley values, converges to the common equilibrium $z_{\ell}^{*}=z_{r}^{*}$ for all $r, \ell \in \mathcal{V}$.

Proof: The proof is presented in the Appendix.

Finally, notice that similar to the population-games approach, the cooperative approach neither requires information about the model of the system. Therefore, the cooperative game is also data-driven.

\section{Case Study: Water Distribution Network}

As a case study, a multi-objective problem involving both competition and cooperation is presented. The case study is shown in Figure 5, which is composed of $N=13$ tanks. There are two control objectives. The former objective is to maintain all the tanks at the same maximum level, which is solved by finding a Nash equilibrium. The latter

3 The proper topology is determined by considering a fair distribution of costs given by the Shapley value, which establishes the appropriate partitioning within the system at every time $\tau$ that satisfies Remark 2 . 


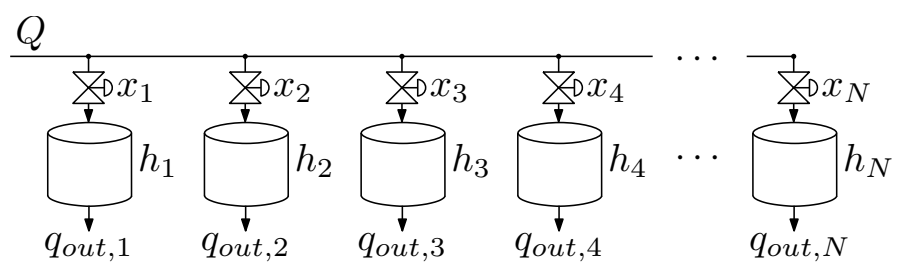

Fig. 5. Case study: Resource $Q$ allocation throughout $N$ tanks.

objective is to determine the cost each player should pay for these communication links according to its contribution to achieve the desired equilibrium. The fair cost that each player should pay is given by the Shapley value.

Let $\varphi_{\text {links }}$ denote the communication cost associated to the undirected graph $\mathcal{G}$ (see Figure 1(b)). Therefore, the fair cost distribution is given by the Shapley value, i.e.,

$$
L_{\ell}=\frac{\Phi(\ell)}{\sum_{r=1}^{N} \Phi(r)} \varphi_{\text {links }}, \ell \in \mathcal{V}
$$

where $L_{\ell}$ is the fair cost corresponding to the player $\ell \in \mathcal{V}$. The Shapley value is normalized in (11) since it is computed in terms of the error rather than in economical units. These communication links allow sharing, in a local way, information about the measured levels in order to achieve the first control objective. Notice that this is a decentralized control scheme since each control input is computed by using only partial and local information.

In order to solve the control problem, it is proposed to make a partitioning based on the Shapley value as presented in Section 4. Therefore, the individual cost associated to each player is the error between the current level and the maximum level of each tank, i.e.,

$$
C_{\ell}=h_{\max , \ell}-h_{\ell},
$$

where $h_{\max , \ell}$ denotes the maximum and constant possible level for the $\ell^{\text {th }}$ tank, and $h_{l}$ is the current measured level of the $\ell^{\text {th }}$ tank. Once the partitions are determined, a population-dynamics approach is applied to each partition, where the fitness function for each strategy is given by the error defined according to Proposition 2, i.e., $f_{\ell}=h_{\max , \ell}-h_{\ell}$, for all $\ell \in \mathcal{V}$. It is assumed that this fitness function is always non-negative since physically the current measured level is $h_{\ell} \leq h_{\max , \ell}$. Notice that, with this fitness function, more resource is assigned to those tanks with lower level. The dynamics for the $\ell^{\text {th }}$ tank are given by

$$
\begin{aligned}
\dot{h}_{\ell} & =q_{\text {in }, \ell}-q_{\text {out }, \ell}, \\
q_{\text {out }, \ell} & =K_{\ell} h_{\ell},
\end{aligned}
$$

where $h_{\ell}$ is the current level, $q_{i n, \ell}$ is the inflow, $q_{\text {out }, \ell}$ is the outflow, and $K_{\ell}$ is a constant factor characterizing the outflow, for the $\ell^{\text {th }}$ tank. There is a constant available resource given by $Q=30 \mathrm{~m}^{3} / \mathrm{s}$. Each inflow $q_{\text {in }, \ell}$ is controlled by a valve commanded by a control signal $x_{\ell}$, i.e., $q_{i n, \ell}=Q x_{\ell}$, with $0 \leq x_{\ell} \leq 1$. It is assumed that there is a local controller at each valve that guarantees the desired flow given by $x_{\ell}$. The limited resource establishes a constraint over all the inflows, i.e., $\sum_{\ell=1}^{N} q_{i n, \ell}=Q$. This equality constraint leads to the condition $\sum_{\ell=1}^{N} Q x_{\ell}=Q$, and consequently, $\sum_{\ell=1}^{N} x_{\ell}=1$, then $\mathbf{x} \in \Delta$. This condition is also satisfied in the partitioned system if the initial condition of the proportion of agents satisfies $\sum_{j \in \mathcal{P}_{i}} \mathbb{1}_{N_{i}^{j}}^{\top} \mathbf{x}_{i}^{j}(0)=1$, for the initial topology $i \in \mathcal{T}$, due to Proposition 1.

In order to analyze the performance of the closed-loop system composed of the flow-based distribution network and the population dynamics, three Key Performance Indicators (KPIs) are stated.

- The costs associated to the communication links $\varphi_{\text {links }}$ is taken as a KPI given by

$$
\varphi_{\text {links }}=\frac{1}{2} \int_{t_{0}}^{t_{f}} \mathbb{1}_{N}^{\top} \mathbf{A}_{i} \mathbb{1}_{N} d t, i \in \mathcal{T}
$$

for the current topology $i \in \mathcal{T}$, where $t_{0}$ is an initial time and $t_{f}$ a final time. 
- The error of each tank level with respect to the average levels of the system tanks is also considered as a performance indicator, i.e.,

$$
\varphi_{\text {error }, \ell}=\int_{t_{0}}^{t_{f}}\left(h_{\ell}-\frac{1}{N} \mathbf{h}^{\top} \mathbb{1}_{N}\right) d t, \ell \in \mathcal{V}
$$

where $\mathbf{h}=\left[\begin{array}{lll}h_{1} & \cdots & h_{N}\end{array}\right]^{\top}$. Then, there is a KPI for the whole system in function of the mentioned error levels, i.e.,

$$
\varphi_{\mathrm{error}}=\sum_{\ell=1}^{N} \varphi_{\mathrm{error}, \ell}
$$

- The settling time of the system states (level of tanks) with criterion of the $5 \%$, i.e.,

$$
\varphi_{\text {settling }}=\min _{t}\{t: \tilde{t} \geq t, \underline{\mathbf{h}} \leq \mathbf{h}(\tilde{t}) \leq \overline{\mathbf{h}}\}
$$

where $\underline{\mathbf{h}}=0.95 \mathbf{h}^{*}, \overline{\mathbf{h}}=1.05 \mathbf{h}^{*}$, with $\mathbf{h}^{*}$ corresponds to the equilibrium point of the system.

\subsection{Results}

As a reference to analyze the performance of the proposed control strategy, results for the centralized case are also presented. This is equivalent to the case with a topology given by a complete graph (see Figure 6).

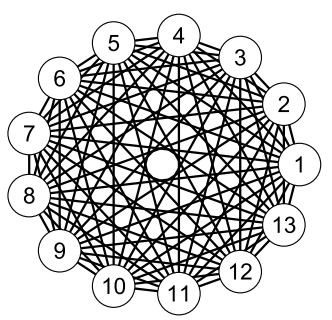

Fig. 6. Complete graph given by the grand coalition.

For this example, $\tau=2.5 \mathrm{~s}$, and the Shapley value is computed as a function of the error costs in (12). It is important to mention that the initial condition for each tank level has been established as a random value from the interval $[0,1]$. The objectives are: i) maintain all the tank levels at the same maximum value; and ii) determine the fair costs for the players.

Figure 7 shows the evolution of the tank levels for three different cases (partitions of 2 players, partitions of 3 players and the grand partition), where it is also shown the gap that determines the settling time. Every $\tau$, the topology of the system is determined. In Figure 7, it can be also seen that the first objective is met for all the cases. The second objective is achieved by determining the Shapley value as presented in Table 2. Figure 8 shows some different topologies obtained based on the Shapley value (see Algorithm 1) at five time instants. In that figure, it can be seen how topologies vary dynamically with partitions of two and three players.

Figure 9 shows a sensitivity analysis for the evolution of the tank levels for $N_{s}=10$ different scenarios. The only difference among these ten scenarios is the vector of initial conditions, which are selected randomly within the interval $[0,2]$. First, it is presented the mean of all the tank levels for the scenarios, i.e.,

$$
\varrho(h(w \delta))=\frac{1}{N_{s} N} \sum_{s=1}^{N_{s}} \sum_{i=1}^{N} h_{i}^{s}(w \delta),
$$

where $w=1, \ldots, 2000$, are the data used to make the sensitivity analysis, $\delta=0.1 \mathrm{~s}$ in order to cover the whole simulation time (i.e., $t_{f}=200 \mathrm{~s}$ ), and $h_{i}^{s}$ corresponds to the tank $i \in \mathcal{V}$ in the $s^{t h}$ simulation. Then, the standard 


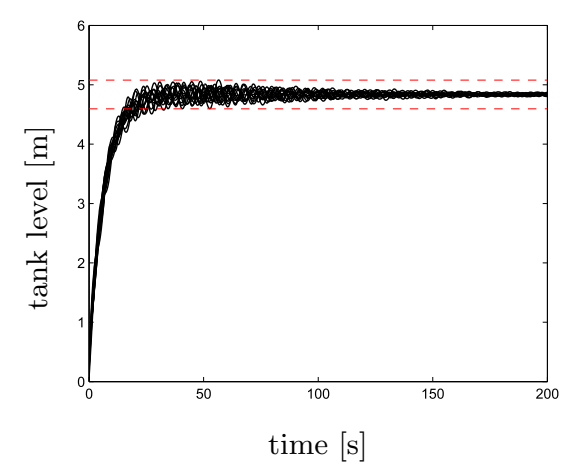

(a)

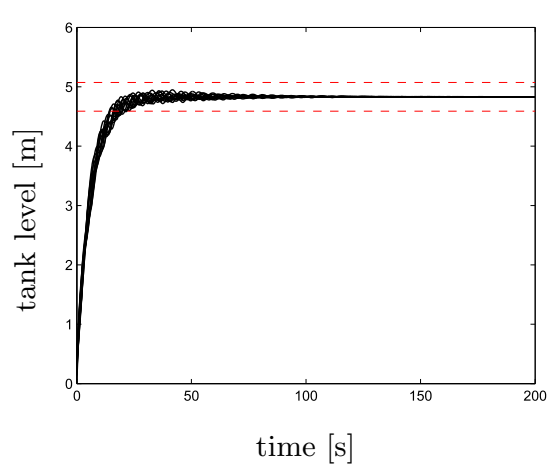

(b)

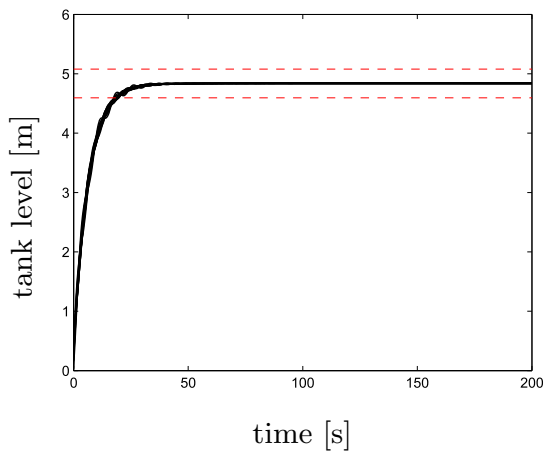

(c)

Fig. 7. Evolution of the 13 tank levels for three different cases: (a) partitions of two players, (b) partitions of three players, and (c) partition of $N$ players (full information). Initial condition for each tank level has been determined by a random value within the interval $[0,1]$.
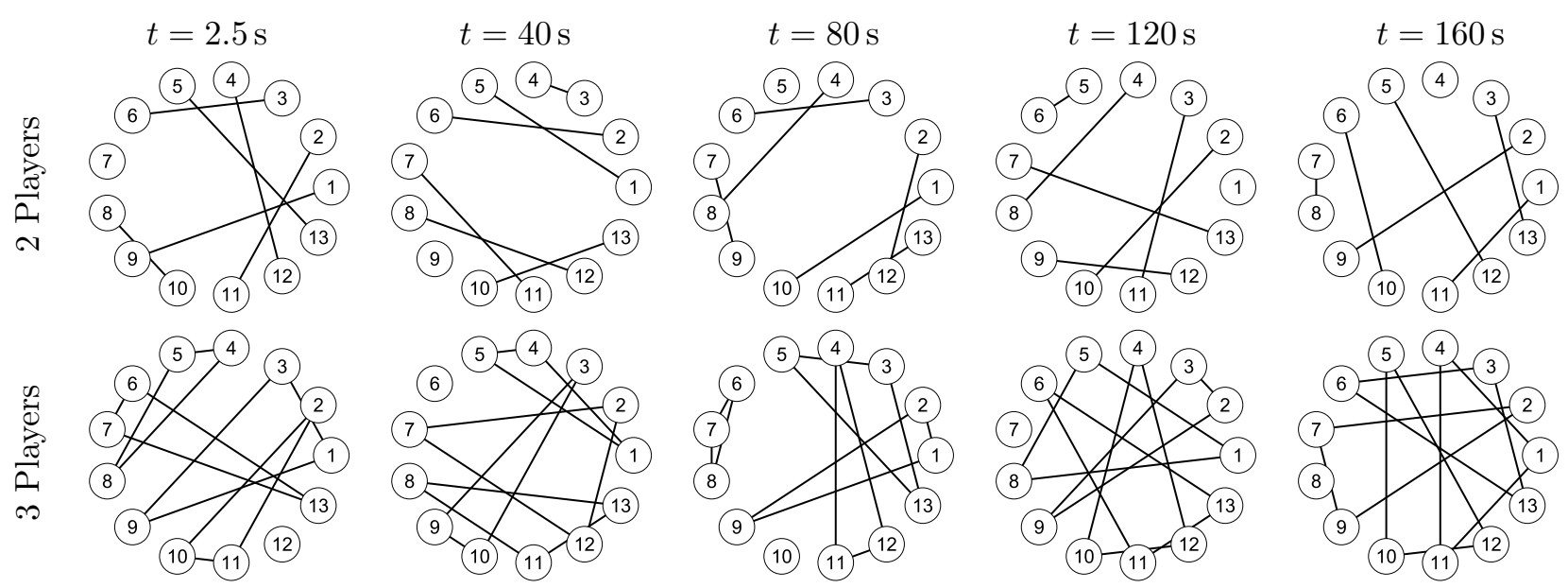

Fig. 8. Evolution of the graph topologies given by partitions of two and three players (i.e., $g=2$, and $g=3$ ), for five different iterations.

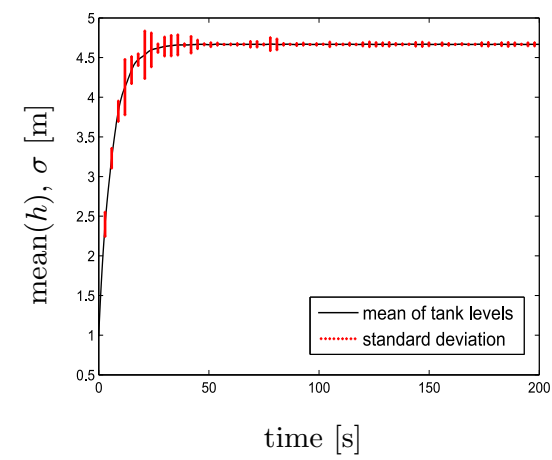

(a)

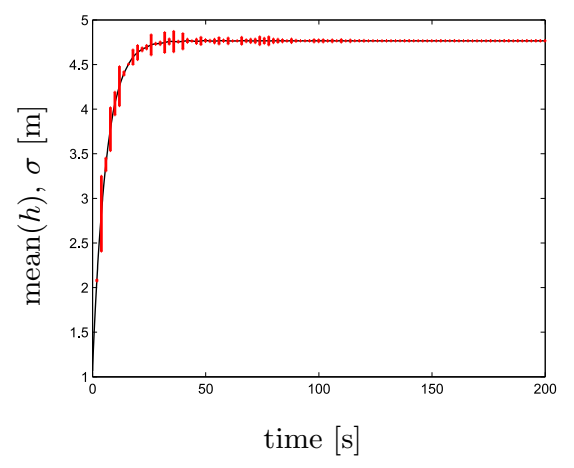

(b)

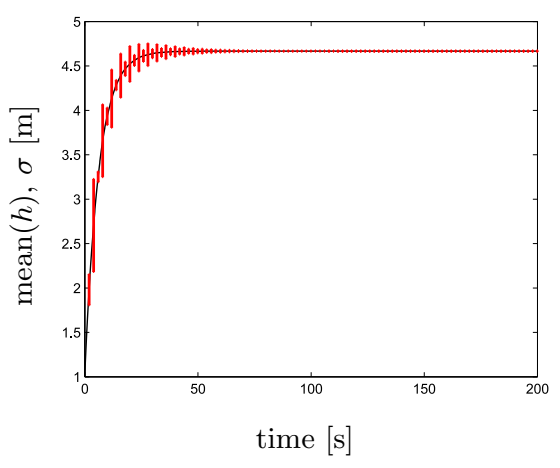

(c)

Fig. 9. Sensitivity analysis made based on scenarios with random initial conditions with $N=13$ tank levels. Mean evolution of the tank levels and their corresponding standard deviation along the time for three different cases: (a) partitions of two players, (b) partition of three players, and (c) partition of $N$ players (full information).

deviation throughout time is presented to analyze the transitory event for each one of the three cases, partitions of 
Table 2

Fair economical costs for each player determined with the Shapley value.

\begin{tabular}{c||cc}
$\begin{array}{c}\text { Player } \\
\ell \in \mathcal{V}\end{array}$ & $\begin{array}{c}\text { Fair costs according Shapley value } \\
\left(\Phi(\ell) \varphi_{\text {links }}\right) /\left(\sum_{r=1}^{N} \Phi(r)\right)\end{array}$ \\
\hline \hline \multirow{2}{*}{1} & Partitions 2 Players & Partitions 3 Players \\
2 & 101.9982 & 162.7683 \\
3 & 103.4772 & 164.7666 \\
4 & 103.0618 & 175.4056 \\
5 & 67.3064 & 161.5046 \\
6 & 69.9416 & 294.2980 \\
7 & 69.7096 & 146.2820 \\
8 & 78.9709 & 215.1184 \\
9 & 123.0623 & 167.3544 \\
10 & 91.6612 & 290.9280 \\
11 & 76.7914 & 146.8018 \\
12 & 99.1411 & 154.4804 \\
13 & 75.6172 & 170.1767 \\
\hline Total & 139.2561 & 150.1145 \\
\hline
\end{tabular}

two players, three players, and the grand partition. The standard deviation is computed as follows:

$$
\sigma(w \delta)=\sqrt{\frac{1}{N_{s} N} \sum_{s=1}^{N_{s}} \sum_{i=1}^{N}\left(h_{i}^{s}(w \delta)-\varrho(h(w \delta))\right)^{2}} .
$$

It can be seen in Figure 9 that the standard deviation is smaller as there is an evolution of the tank levels. Besides, the standard deviations are reduced more quickly when there are larger partitions. This fact is because when having bigger partitions, there are more communication exchange among strategies in the population game achieving the closed-loop system equilibrium in a faster way. The graph sequence presented in Figure 8 varies as the initial conditions are modified because the Shapley value depends on them. However, the sensitivity analysis presented in Figure 9 shows that the system achieves the equilibrium point for all the different initial conditions.

Finally, the comparison of the three cases with the proposed KPI is presented in Table 3 . In these results, it can be seen the proper performance of the proposed methodology with respect to the centralized case with full information (partition of $N$ players). Economical costs are reduced significantly, but it implies an increment of the transitory errors and settling time of the system states (tank levels).

Table 3

Closed-loop system performance for different topologies. Coalitions of two and three players, and with full information.

\begin{tabular}{c||ccc}
$\begin{array}{c}\text { Players per } \\
\text { partitions } g\end{array}$ & $\begin{array}{c}\text { Communication } \\
\varphi_{\text {links }}\end{array}$ & $\begin{array}{c}\text { Error } \\
\varphi_{\text {error }}\end{array}$ & $\begin{array}{c}\text { Settling time } \\
\varphi_{\text {settling }}\end{array}$ \\
\hline \hline 2 players & 1200 & 197.1742 & 33.23 \\
3 players & 2400 & 58.1723 & 21.80 \\
$N$ players & 15600 & 20.9294 & 19.73 \\
\hline
\end{tabular}

\subsection{Discussion}

In general, game theory models the interaction between rational players. The two different main approaches, the cooperative and the non-cooperative game directions, solve quite different problems. One approach addresses problems in which there is competition whereas the other one is more appropriate to model cooperation. As it has been 
seen here, it is possible to face engineering applications by implementing both approaches in different but related objectives.

The combination of both approaches demands that the elements within the system take different roles. For instance, in the presented flow-based distribution network, storage nodes represent strategies in the non-cooperative game approach. In contrast, storage nodes assume the role of players that demand information by using the communication network in the cooperative game. This multi-role situation is quite interesting due to the fact that each role is tightly related to each particular control objective, i.e., the optimal resource distribution for the non-cooperative game and both the fair distribution of costs and proper partitioning for the cooperative game.

It has been shown that the closed-loop system with the non-cooperative approach (i.e., with both the replicator and the projection dynamics) is stable, performing the partitioning criteria based on cooperative games. This is achieved based on the assumption that all the partitions, for all the topologies, form a complete graph. Aditionally, when selecting conveniently the characteristic function of the cooperative game, it is possible to compute the Shapley value of the whole set of players from the Shapley value of any arbitrary player by following a polynomial-time procedure. Moreover, it has been shown that, by taking advantage of the coalition rationality axiom, it is possible to compute the Shapley value by solving a set of linear equations. This result allows to reduce considerably the computational burden from an exponential-time procedure to a polynomial one. Besides, due to the fact that the computation can be reduced, it is also possible to spend time to compute the Shapley value under a distributed structure. For example, the comparison of the computational burden in Table 1 shows less computation cost, for the distributed structure with respect to the traditional Shapley equation and a case involving more than ten players.

\section{Conclusions}

A control problem involving two objectives associated to competition and cooperation has been presented. In particular, a flow-based distribution network composed of different sub-systems (storage nodes) has been considered. The first objective is associated to an optimal distribution of the available resource, i.e., it is desired to achieve a maximum and equal amount of resource throughout all the sub-systems (storage nodes). To this end, a communication network allows the interaction among different storage nodes, whose communication links have an economical cost. As a second objective, it is desired to distribute the costs of the communication network throughout the subsystems, where more influent tanks (with higher power index) must pay less than those with lower influence (with lower power index), i.e., it is desired to assign fair costs for each storage node according to their contributions to the achievement of the first objective. A non-cooperative-game approach is used to solve the former objective, whereas a cooperative-game approach is proposed to solve the latter objective.

Furthermore, for the non-cooperative-game approach, the stability of the closed-loop system is analyzed to guarantee that the first objective is achieved. On the other hand, regarding the cooperative-game approach that determines the fair distribution of communication costs, an alternative way to compute the Shapley value for the selected specific characteristic function has been presented in order to mitigate the computation burden. The proposed approach also allows the computation of the Shapley value under distributed structures within reasonable computational times with respect to the standard computation.

Moreover, bigger partitions imply more information exchange among the storage nodes, and therefore the resource distribution objective is achieved faster. Simulation results have shown that the steady state is achieved faster as the number of players in the partitions is greater. When having large partitions, the dynamic resource allocation is made faster due to the fact that there is more available information in the non-cooperative game approach. Nevertheless, when having more players making partitions, more communication links are required and this implies higher economical costs. Furthermore, a smaller overshoot and settling time for the system states (tank levels) are obtained when partitions are larger. Hence, there is a need for balancing economical cost and performance in relation to the complexity of the system and the number of players involved in the cooperative game to perform the partitioning. Finally, the sensitivity analysis for the evolution of the system states with arbitrary initial conditions shows that, despite the fact that the partitioning highly depends on the initial conditions of the system, the equilibrium is always achieved. 


\section{Appendix}

Proof of Lemma 1: The storage model dynamics in error coordinates $\dot{e}_{z \ell}$ are as follows:

$$
\dot{e}_{z_{\ell}}=Q\left(e_{x_{\ell}}+x_{\ell}^{*}\right)-K_{\ell}\left(e_{z_{\ell}}+z_{\ell}^{*}\right), \text { for all } \ell \in \mathcal{V}_{i}^{j} .
$$

Then, consider the storage function (also Lyapunov function)

$$
L_{1}=\frac{1}{2 Q} \sum_{i \in \mathcal{T}} \sum_{j \in \mathcal{P}_{i}} \sum_{\ell \in \mathcal{V}_{i}^{j}} e_{z_{\ell}}^{2},
$$

whose derivative is given by

$$
\begin{aligned}
\dot{L}_{1} & =\frac{1}{Q} \sum_{i \in \mathcal{T}} \sum_{j \in \mathcal{P}_{i}} \sum_{\ell \in \mathcal{V}_{i}^{j}} e_{z_{\ell}} \dot{e}_{z_{\ell}}, \\
& =\frac{1}{Q} \sum_{i \in \mathcal{T}} \sum_{j \in \mathcal{P}_{i}} \sum_{\ell \in \mathcal{V}_{i}^{j}} e_{z_{\ell}} Q\left(e_{x_{\ell}}+x_{\ell}^{*}\right)-e_{z_{\ell}} K_{\ell}\left(e_{z_{\ell}}+z_{\ell}^{*}\right), \\
& =\frac{1}{Q} \sum_{i \in \mathcal{T}} \sum_{j \in \mathcal{P}_{i}} \sum_{\ell \in \mathcal{V}_{i}^{j}} e_{z_{\ell}} Q e_{x_{\ell}}+e_{z_{\ell}} Q x_{\ell}^{*}-e_{z_{\ell}}^{2} K_{\ell}-e_{z_{\ell}} K_{\ell} z_{\ell}^{*}, \\
& =\frac{1}{Q} \sum_{i \in \mathcal{T}} \sum_{j \in \mathcal{P}_{i}} \sum_{\ell \in \mathcal{V}_{i}^{j}} e_{z_{\ell}} Q e_{x_{\ell}}-e_{z_{\ell}}^{2} K_{\ell}+e_{z_{\ell}} \underbrace{\left(Q x_{\ell}^{*}-K_{\ell} z_{\ell}^{*}\right)}_{\dot{z}_{\ell}^{*}=0}, \\
& \leq \sum_{i \in \mathcal{T}} \sum_{j \in \mathcal{P}_{i}} \sum_{\ell \in \mathcal{V}_{i}^{j}} e_{z_{\ell}} e_{x_{\ell}}, \\
& =\sum_{i \in \mathcal{T}} \sum_{j \in \mathcal{P}_{i}} \sum_{\ell \in \mathcal{V}_{i}^{j}}\left(z_{\ell}-z_{\ell}^{*}\right)\left(x_{\ell}-x_{\ell}^{*}\right), \\
& =\sum_{i \in \mathcal{T}} \sum_{j \in \mathcal{P}_{i}}\left(\mathbf{z}_{i}^{j}-\mathbf{z}_{i}^{j *}\right)^{\top}\left(\mathbf{x}_{i}^{j}-\mathbf{x}_{i}^{j *}\right) .
\end{aligned}
$$

Then, since $\left(\mathbf{z}_{i}^{j}-\mathbf{z}_{i}^{j *}\right)$ are the inputs and $\left(\mathbf{x}_{i}^{j}-\mathbf{x}_{i}^{j *}\right)$ are the outputs, then $\dot{L}_{1} \leq \sum_{i \in \mathcal{T}} \sum_{j \in \mathcal{P}_{i}}\left(\mathbf{z}_{i}^{j}-\mathbf{z}_{i}^{j *}\right)^{\top}\left(\mathbf{x}_{i}^{j}-\mathbf{x}_{i}^{j *}\right)$ allows to conclude that storage-node dynamics in the flow-based distribution network are passive.

Proof of Lemma 2: The analysis is made for both replicator and projection dynamics using different storage functions. Replicator dynamics: consider the entropy function as a storage function (also Lyapunov function)

$$
\begin{aligned}
L_{2}(\mathbf{x}) & =-\sum_{i \in \mathcal{T}} \sum_{j \in \mathcal{P}_{i}} \sum_{\ell \in \mathcal{V}_{i}^{j}} x_{i, \ell}^{j *} \ln \left(\frac{x_{i, \ell}^{j}}{x_{i, \ell}^{j *}}\right), \\
& =-\sum_{i \in \mathcal{T}} \sum_{\ell \in \mathcal{V}} x_{l}^{*} \ln \left(\frac{x_{\ell}}{x_{\ell}^{*}}\right),
\end{aligned}
$$

which is a valid Lyapunov function since $L\left(\mathbf{x}^{*}\right)=0$ and $L(\mathbf{x})>0$, for all $\mathbf{x} \neq \mathbf{x}^{*}$, fact that is verified by using the Jensen's inequality (i.e., $E(g(x)) \geq g(E(x))$ for any convex function as the logarithm [13]), e.g., $L(\mathbf{x}) \geq$ $-\sum_{i \in \mathcal{T}} \ln \left(\sum_{\ell \in \mathcal{V}} x_{\ell}^{*} \frac{x_{\ell}}{x_{\ell}^{*}}\right)$, and due to the fact that $\ln \left(\sum_{\ell \in \mathcal{V}} x_{\ell}^{*} \frac{x_{\ell}}{x_{\ell}^{*}}\right)=\ln (1)$, it follows that $L(\mathbf{x}) \geq 0$. Then $L(\mathbf{x})>0$, for all $\mathbf{x} \neq \mathbf{x}^{*}$. Hence,

$$
\dot{L}_{2}(\mathbf{x})=-\sum_{i \in \mathcal{T}} \sum_{j \in \mathcal{P}_{i}} \sum_{\ell \in \mathcal{V}_{i}^{j}} \frac{x_{i, \ell}^{j *}}{x_{i, \ell}^{j}} \dot{x}_{i, \ell}^{j} .
$$


Replacing dynamics (2) in (16) yields

$$
\begin{aligned}
\dot{L}_{2}(\mathbf{x}) & =-\sum_{i \in \mathcal{T}} \sum_{j \in \mathcal{P}_{i}} \mathbf{x}_{i}^{j^{* \top}}\left(\mathbf{f}_{i}^{j}-\frac{1}{m_{i}^{j}} \mathbb{1}_{N_{i}^{j}} \mathbf{x}_{i}^{j^{\top}} \mathbf{f}_{i}^{j}\right), \\
& =-\sum_{i \in \mathcal{T}} \sum_{j \in \mathcal{P}_{i}}\left(\mathbf{x}_{i}^{j^{* \top}}-\frac{1}{m_{i}^{j}} \mathbf{x}_{i}^{j^{* \top}} \mathbb{1}_{N_{i}^{j}} \mathbf{x}_{i}^{j^{\top}}\right) \mathbf{f}_{i}^{j}, \\
& =\sum_{i \in \mathcal{T}} \sum_{j \in \mathcal{P}_{i}}\left(\mathbf{x}_{i}^{j}-\mathbf{x}_{i}^{j^{*}}\right)^{\top} \mathbf{f}_{i}^{j} .
\end{aligned}
$$

Projection dynamics: consider the quadratic error as a storage function (also Lyapunov function), i.e.,

$$
L_{3}(\mathbf{x})=\frac{1}{2} \sum_{i \in \mathcal{T}} \sum_{j \in \mathcal{P}_{i}} \sum_{\ell \in \mathcal{V}_{i}^{j}}\left(x_{i, \ell}^{j}-x_{i, \ell}^{j *}\right)^{2},
$$

where $L(\mathbf{x})>0$ for all $\mathbf{x} \neq \mathbf{x}^{*}$, and $L\left(\mathbf{x}^{*}\right)=0$. Replacing dynamics (3) in the storage function derivative yields

$$
\begin{aligned}
\dot{L}_{3}(\mathbf{x}) & =\sum_{i \in \mathcal{T}} \sum_{j \in \mathcal{P}_{i}}\left(\mathbf{x}_{i}^{j}-\mathbf{x}_{i}^{j *}\right)^{\top}\left(\mathbf{f}_{i}^{j}-\frac{1}{N_{i}^{j}} \mathbb{1}_{N_{i}^{j}} \mathbf{f}_{i}^{j^{\top}} \mathbb{1}_{N_{i}^{j}}\right), \\
& =\sum_{i \in \mathcal{T}} \sum_{j \in \mathcal{P}_{i}}\left(\mathbf{x}_{i}^{j}-\mathbf{x}_{i}^{j *}\right)^{\top} \mathbf{f}_{i}^{j}-\frac{1}{N_{i}^{j}}\left(\mathbf{x}_{i}^{j}-\mathbf{x}_{i}^{j *}\right)^{\top} \mathbb{1}_{N_{i}^{j}} \mathbf{f}_{i}^{j^{\top}} \mathbb{1}_{N_{i}^{j}}, \\
& =\sum_{i \in \mathcal{T}} \sum_{j \in \mathcal{P}_{i}}\left(\mathbf{x}_{i}^{j}-\mathbf{x}_{i}^{j *}\right)^{\top} \mathbf{f}_{i}^{j}-\frac{1}{N_{i}^{j}}\left(\mathbf{x}_{i}^{j^{\top}} \mathbb{1}_{N_{i}^{j}}-\mathbf{x}_{i}^{j *}{ }^{\top} \mathbb{1}_{N_{i}^{j}}\right) \mathbf{f}_{i}^{j^{\top}} \mathbb{1}_{N_{i}^{j}}, \\
& =\sum_{i \in \mathcal{T}} \sum_{j \in \mathcal{P}_{i}}\left(\mathbf{x}_{i}^{j}-\mathbf{x}_{i}^{j *}\right)^{\top} \mathbf{f}_{i}^{j} .
\end{aligned}
$$

Then, since $\mathbf{f}_{\mathbf{i}}^{\mathbf{j}}$ are the inputs and $\left(\mathbf{x}_{i}^{j}-\mathbf{x}_{i}^{j *}\right)$ are the outputs, both results show that the replicator and projection dynamics are lossless.

Proof of Lemma 3: Consider the arbitrary initial condition for the storage nodes $\mathbf{z}(0) \in \mathbb{R}_{\geq 0}^{N}$, the maximum capacities for the storage nodes $\mathbf{z}_{\max } \in \mathbb{R}_{\geq 0}^{N}$, and the costs $C_{i}(0)=z_{\max , i}-z_{i}(0)$, for all $i \in \mathcal{V}$. Now, consider $r^{0}=\arg \max _{i \in \mathcal{V}} C_{i}(0)$, and $\ell^{0}=\arg \min _{i \in \mathcal{V}} C_{i}(0)$. Then, it is known that according to Remark $3, \Phi\left(r^{0}\right)>\Phi\left(\ell^{0}\right)$ and that $r^{0}, \ell^{0} \in \mathcal{V}$ are assigned to the same partition according to the proposed partitioning procedure (Algorithm 1). It follows that, during the interval time $\tau$ with the closed loop presented in Figure 3 and according to Remark 2, it is obtained $C_{\ell^{0}} \leq z_{\max , r^{0}}-z_{r^{0}}(\tau) \leq C_{r^{0}}$, and $C_{\ell^{0}} \leq z_{\max , r^{0}}-z_{r^{0}}(\tau) \leq C_{r^{0}}$. Then computing $r^{1}=\arg \max _{i \in \mathcal{V}} C_{i}(\tau)$, and $\ell^{1}=\arg \min _{i \in \mathcal{V}} C_{i}(\tau)$, it is obtained that $C_{\ell^{0}} \leq C_{\ell^{1}}$, and $C_{r^{1}} \leq C_{r^{0}}$.

Making the partitioning, it follows that $C_{\ell^{0}} \leq C_{\ell^{1}} \leq z_{\max , \ell^{1}}-z_{\ell^{1}}(2 \tau) \leq C_{r^{1}} \leq C_{r^{0}}$, and $C_{\ell^{0}} \leq C_{\ell^{1}} \leq z_{\max , r^{1}}-$ $z_{r^{1}}(2 \tau) \leq C_{r^{1}} \leq C_{r^{0}}$. It is concluded that

$$
\begin{aligned}
& \min _{i \in \mathcal{V}} C_{i}(k \tau) \leq z_{\max , \ell}-z_{\ell}((k+1) \tau) \leq \max _{i \in \mathcal{V}} C_{i}(k \tau), \\
& \min _{i \in \mathcal{V}} C_{i}(k \tau) \leq z_{\max , r}-z_{r}((k+1) \tau) \leq \max _{i \in \mathcal{V}} C_{i}(k \tau),
\end{aligned}
$$

where $r=\arg \max _{i \in \mathcal{V}} C_{i}(k \tau)$, and $\ell=\arg \min _{i \in \mathcal{V}} C_{i}(k \tau)$. Then $\left[\max _{i \in \mathcal{V}} C_{i}(k \tau)-\min _{i \in \mathcal{V}} C_{i}(k \tau)\right] \rightarrow 0$ as $k \rightarrow \infty$, until $\max _{i \in \mathcal{V}} C_{i}(k \tau)=\min _{i \in \mathcal{V}} C_{i}(k \tau)$. This situation leads to the equilibrium $z_{r}^{*}=z_{\ell}^{*}$, for all $r, \ell \in \mathcal{V}$. Consequently, it is obtained that all fitness functions are the same, then $x_{\ell}^{*}$ is achieved for all $\ell \in \mathcal{V}$.

Proof of Proposition 1: The invariant-set property is analyzed for both population dynamics. 
Replicator dynamics: in order to prove that the simplex $\Delta$ is an invariant set, it is shown that the sum of $\dot{\mathbf{x}}_{i}^{j}$ for all topologies $i \in \mathcal{T}$ and for all partitions $j \in \mathcal{P}_{i}$ is null:

$$
\begin{aligned}
\sum_{i \in \mathcal{T}} \sum_{j \in \mathcal{P}_{i}} \mathbb{1}_{N_{i}^{j}}^{\top} \dot{\mathbf{x}}_{i}^{j} & =\sum_{i \in \mathcal{T}} \sum_{j \in \mathcal{P}_{i}} \mathbf{x}_{i}^{j^{\top}}\left(\mathbf{f}_{i}^{j}-\frac{1}{m_{i}^{j}} \mathbb{1}_{N_{i}^{j}} \mathbf{x}_{i}^{j^{\top}} \mathbf{f}_{i}^{j}\right) \\
& =\sum_{i \in \mathcal{T}} \sum_{j \in \mathcal{P}_{i}}\left(\mathbf{x}_{i}^{j^{\top}} \mathbf{f}_{i}^{j}-\frac{1}{m_{i}^{j}} \mathbf{x}_{i}^{j^{\top}} \mathbb{1}_{N_{i}^{j}} \mathbf{x}_{i}^{j^{\top}} \mathbf{f}_{i}^{j}\right) \\
& =\sum_{i \in \mathcal{T}} \sum_{j \in \mathcal{P}_{i}}\left(\mathbf{x}_{i}^{j^{\top}} \mathbf{f}_{i}^{j}-\mathbf{x}_{i}^{j^{\top}} \mathbf{f}_{i}^{j}\right), \\
& =0 .
\end{aligned}
$$

Projection dynamics: in order to prove that the simplex $\Delta$ is an invariant set, it is shown that the sum of $\dot{\mathbf{x}}_{i}^{j}$ for all topologies $i \in \mathcal{T}$ and for all partitions $j \in \mathcal{P}_{i}$ is null:

$$
\begin{aligned}
\sum_{i \in \mathcal{T}} \sum_{j \in \mathcal{P}_{i}} \mathbb{1}_{N_{i}^{j}}^{\top} \dot{\mathbf{x}}_{i}^{j} & =\sum_{i \in \mathcal{T}} \sum_{j \in \mathcal{P}_{i}} \mathbb{1}_{N_{i}^{j}}^{\top}\left(\mathbf{f}_{i}^{j}-\frac{1}{N_{i}^{j}} \mathbb{1}_{N_{i}^{j}} \mathbf{f}_{i}^{j^{\top}} \mathbb{1}_{N_{i}^{j}}\right) \\
& =\sum_{i \in \mathcal{T}} \sum_{j \in \mathcal{P}_{i}} \mathbb{1}_{N_{i}^{j}}^{\top} \mathbf{f}_{i}^{j}-\frac{1}{N_{i}^{j}} \mathbb{1}_{N_{i}^{j}}^{\top} \mathbb{1}_{N_{i}^{j}} \mathbf{f}_{i}^{j} \mathbb{1}_{N_{i}^{j}}
\end{aligned}
$$

Notice that $\frac{1}{N_{i}^{j}} \mathbb{1}_{N_{i}^{j}}^{\top} \mathbb{1}_{N_{i}^{j}}=1$, then

$$
\begin{aligned}
\sum_{i \in \mathcal{T}} \sum_{j \in \mathcal{P}_{i}} \mathbb{1}_{N_{i}^{j}}^{\top} \dot{\mathbf{x}}_{i}^{j} & =\sum_{i \in \mathcal{T}} \sum_{j \in \mathcal{P}_{i}} \mathbb{1}_{N_{i}^{j}}^{\top} \mathbf{f}_{i}^{j}-\mathbf{f}_{i}^{j^{\top}} \mathbb{1}_{N_{i}^{j}} \\
& =0 .
\end{aligned}
$$

Both results complete the proof.

Proof of Proposition 2: Consider the Lyapunov function given by $L=L_{1}+L_{2}$ for the replicator dynamics, and $L=L_{1}+L_{3}$ for the projection dynamics, where $L_{1}, L_{2}$ and $L_{3}$ are the functions presented in (14), (15), and (17), respectively. According to Lemmas 1 and 2, for both cases $\dot{L}$ is as follows:

$$
\dot{L} \leq \sum_{i \in \mathcal{T}} \sum_{j \in \mathcal{P}_{i}}\left(\mathbf{z}_{i}^{j}-\mathbf{z}_{i}^{j *}\right)^{\top}\left(\mathbf{x}_{i}^{j}-\mathbf{x}_{i}^{j *}\right)+\sum_{i \in \mathcal{T}} \sum_{j \in \mathcal{P}_{i}}\left(\mathbf{x}_{i}^{j}-\mathbf{x}_{i}^{j *}\right)^{\top} \mathbf{f}_{i}^{j} .
$$

Adding and substracting $\sum_{i \in \mathcal{T}} \sum_{j \in \mathcal{P}_{i}} \mathbf{z}_{\max , i}^{j}$ in (18) yields

$$
\begin{aligned}
\dot{L} & \leq \sum_{i \in \mathcal{T}} \sum_{j \in \mathcal{P}_{i}}\left(-\mathbf{z}_{\max , i}^{j}+\mathbf{z}_{i}^{j}+\mathbf{z}_{\max , i}^{j}-\mathbf{z}_{i}^{j *}\right)^{\top}\left(\mathbf{x}_{i}^{j}-\mathbf{x}_{i}^{j *}\right)+\sum_{i \in \mathcal{T}} \sum_{j \in \mathcal{P}_{i}}\left(\mathbf{x}_{i}^{j}-\mathbf{x}_{i}^{j *}\right)^{\top} \mathbf{f}_{i}^{j}, \\
& =\sum_{i \in \mathcal{T}} \sum_{j \in \mathcal{P}_{i}}\left\{\left(-\mathbf{z}_{\max , i}^{j}+\mathbf{z}_{i}^{j}\right)^{\top}\left(\mathbf{x}_{i}^{j}-\mathbf{x}_{i}^{j *}\right)+\left(\mathbf{z}_{\max , i}^{j}-\mathbf{z}_{i}^{j *}\right)^{\top}\left(\mathbf{x}_{i}^{j}-\mathbf{x}_{i}^{j *}\right)+\mathbf{f}_{i}^{j^{\top}}\left(\mathbf{x}_{i}^{j}-\mathbf{x}_{i}^{j *}\right)\right\}, \\
& =\sum_{i \in \mathcal{T}} \sum_{j \in \mathcal{P}_{i}}\left\{-\mathbf{f}_{i}^{j^{\top}}\left(\mathbf{x}_{i}^{j}-\mathbf{x}_{i}^{j *}\right)+\mathbf{f}_{i}^{j * \top}\left(\mathbf{x}_{i}^{j}-\mathbf{x}_{i}^{j *}\right)+\mathbf{f}_{i}^{j^{\top}}\left(\mathbf{x}_{i}^{j}-\mathbf{x}_{i}^{j *}\right)\right\}, \\
& =0 .
\end{aligned}
$$

Therefore, the equilibrium point $\left(\mathbf{z}_{i}^{j *}, \mathbf{x}_{i}^{j *}\right)$, for all partitions $j \in \mathcal{P}_{i}$ in the topology $i \in \mathcal{T}$, is stable. 


\section{Acknowledgements}

Authors would like to thank COLCIENCIAS (grant 6172) and Agència de Gestió d'Ajust Universitaris i de Recerca AGAUR (FI 2014) for supporting J. Barreiro-Gomez. Authors also would like to thank the projects DEOCS (Ref. DPI2016-76493-C3-3-R), "Control Predictivo en Red" (Ref. DPI2008-05818) from the Spanish Ministry of Science and Education; and the European project FP7-ICT DYMASOS (grant agreement 611281), which have partially supported this work.

\section{References}

[1] J. Barreiro-Gomez, G. Obando, G. Riaño-Briceño, N. Quijano, and C. Ocampo-Martinez. Decentralized control for urban drainage systems via population dynamics: Bogota case study. In Proceedings of the European Control Conference (ECC), pages 2431-2436, Linz, Austria, 2015.

[2] J. Barreiro-Gomez, C. Ocampo-Martinez, J.M. Maestre, and N. Quijano. Multi-objective model-free control based on population dynamics and cooperative games. In Proceedings of the IEEE Conference on Decision and Control (CDC), pages 5296-5301, Osaka, Japan, 2015.

[3] J. Barreiro-Gomez, N. Quijano, and C. Ocampo-Martinez. Constrained distributed optimization: A population dynamics approach. Automatica, 69:101-116, 2016.

[4] T. Basar and G. J. Olsder. Dynamic Noncooperative Game Theory. Academic Press, London/New York, 1995.

[5] K. Cai and H. Ishii. Average consensus on arbitrary strongly connected digraphs with time-varying topologies. IEEE Transactions on Automatic Control, 59:1066-1071, 2014.

[6] F. Fele, J. M. Maestre, and E. F. Camacho. Coalitional control: Cooperative game theory and control. IEEE Control Systems, 37(1):53-69, 2017.

[7] F. Fele, J. M. Maestre, M. Hashemy, D. Muñoz de la Peña, and E. F. Camacho. Coalitional model predictive control of an irrigation canal. Journal of Process Control, 24:314 - 325, 2014.

[8] M. J. Fox and J. S. Shamma. Population games, stable games, and passivity. Games, 4(4):561-583, 2013.

[9] F. Garin and L. Schenato. A survey on distributed estimation and control applications using linear consensus algorithms. Networked Control Systems, 406:75-107, 2010.

[10] R. Gopalakrishnan, J. Marden, and A. Wierman. Characterizing distribution rules for cost sharing games. In Proceeding of the 5th International Conference on Network Games, Control and Optimization (NetGCooP), pages 1-4, Paris, France, 2011.

[11] J. Grosso. Economic and Robust Operation of Generalised Flow-based Networks. Doctoral dissertation. Universidad Politècnica de Catalunya. Automatic Control Department, 2015.

[12] J. Hofbauer and W. H. Sandholm. Stable games and their dynamics. Journal of Economic Theory, 144(4):1665-1693, 2009.

[13] J. L. W. V. Jensen. Sur les fonctions convexes et les ingalits entre les valeurs moyennes. Acta Mathematica, 30(1):175-193, 1906.

[14] M. Jilg and O. Stursberg. Optimized distributed control and topology design for hierarchically interconnected systems. In Proceedings of the 2013 European Control Conference, pages 4340-4346, Zurich, Switzerland, 2013.

[15] M. A. Khan, H. Tembine, and A. V Vasilakos. Evolutionary coalitional games: design and challenges in wireless networks. IEEE Wireless Communications, 19(2):50-56, 2012.

[16] S. Li, Y. Zhang, and Q. Zhu. Nash-optimization enhanced distributed model predictive control applied to the shell benchmark problem. Information Sciences, 170(2-4):329-349, 2005.

[17] J. M. Maestre and R. R. Negenborn, editors. Distributed Model Predictive Control Made Easy, volume 69 of Intelligent Systems, Control and Automation: Science and Engineering. Springer, 2014.

[18] J.M. Maestre, D. Muñoz de la Peña, A. Jiménez Losada, E. Algaba, and E.F. Camacho. A coalitional control scheme with applications to cooperative game theory. Optimal Control Applications and Methods, 35:592-608, 2014.

[19] J. Marden and J. Shamma. Game theory and distributed control, volume 4, chapter Handbook of Game Theory with Economic Applications, pages 861-899. Elsevier, 2015.

[20] F. Muros, J.M. Maestre, E. Algaba, C. Ocampo-Martinez, and E. F. Camacho. An application of the Shapley value to perform system partitioning. In Proceedings of the American Control Conference (ACC), pages 2143-2148, Chicago, Illinois, 2015.

[21] F. J. Muros Ponce, J. M. Maestre, E. Algaba, T. Alamo, and E. F. Camacho. An iterative design method for coalitional control networks with constraints on the Shapley value. In Proceedings of the 19th IFAC World Congress, pages 1188-1193, Cape Town, South Africa, 2014.

[22] R. B. Myerson. Game Theory. Analysis of Conflict. Hardvard University Press, 1997.

[23] A. Nagurney and D. Zhang. Projected dynamical systems in the formulation, stability analysis, and computation of fixed demand traffic network equilibria. Transportation Science, 31:147-158, 1997.

[24] J. Nash. Equilibrium points in n-person games. Proceedings of the National Academy of Sciences, 36:48-49, 1950.

[25] A. Nuñez, C. Ocampo-Martinez, J.M. Maestre, and B. De Schutter. Time-Varying Scheme for Noncentralized Model Predictive Control of Large-Scale Systems. Mathematical Problems in Engineering, (v2015):1-17, 2015. 
[26] G. Obando, A. Pantoja, and N. Quijano. Building Temperature Control based on Population Dynamics. IEEE Transactions on Control Systems Technology, 22(1):404-412, 2014.

[27] R. Olfati-Saber, J.A. Fax, and R.M. Murray. Consensus and cooperation in networked multi-agent systems. Proceedings of the IEEE, 95(1):215-233, 2007.

[28] G. Owen. Game Theory. Academic Press, 1995.

[29] A. Pantoja and N. Quijano. A population dynamics approach for the dispatch of distributed generators. IEEE Transactions on Industrial Electronics, 58(10):4559-4567, 2011.

[30] A. Pashaie, L. Pavel, and C. J. Damaren. Population dynamics approach for resource allocation problems. In Proceedings of the American Control Conference (ACC), pages 5231-5237, Chicago, IL, USA, 2015.

[31] N. Quijano, C. Ocampo-Martinez, J. Barreiro-Gomez, G. Obando, A. Pantoja, and E. Mojica-Nava. The role of population games and evolutionary dynamics in distributed control systems. IEEE Control Systems, 37(1):70-97, 2017.

[32] E. Ramirez-Llanos and N. Quijano. A population dynamics approach for the water distribution problem. International Journal of Control, 83(9):1947-1964, 2010.

[33] S. Riverso, M. Farina, and G. Ferrari-Trecate. Plug-and-play decentralized model predictive control for linear systems. IEEE Transactions on Automatic Control, 58(10):2608-2614, 2013.

[34] N.R. Sandell, P. Varaiya, M. Athans, and M.G. Safonov. Survey of decentralized control methods for large scale systems. IEEE Transactions on Automatic Control, 23(2):108-128, 1978.

[35] W. H. Sandholm. Population games and evolutionary dynamics. Cambridge, Mass. MIT Press, 2010.

[36] W. H. Sandholm, E. Dokumaci, and R. Lahkar. The projection dynamic and the replicator dynamic. Games and Economic Behavior, 64(2):666-683, 2008.

[37] L.S. Shapley. A value for n-person games. Annals of Mathematics Studies, 28:307-317, 1953.

[38] P. D. Taylor and L. B. Jonker. Evolutionary stable strategies and game dynamics. Mathematical Biosciences, 40(1):145-156, 1978.

[39] H. Tembine, E. Altman, R. El-Azouzi, and Y. Hayel. Evolutionary games in wireless networks. IEEE Transactions on Systems, Man, and Cybernetics, Part B: Cybernetics, 40(3):634-646, 2010. 